\title{
METRIC SPACES WITH UNIQUE PRETANGENT SPACES. CONDITIONS OF THE UNIQUENESS
}

\author{
Fahreddin Abdullayev, Oleksiy Dovgoshey and Mehmet Küçükaslan \\ Mersin University, Faculty of Literature and Science, Department of Mathematics \\ 33342 Mersin, Turkey; fabdul@mersin.edu.tr \\ National Academy of Sciences of Ukraine, Institute of Applied Mathematics and Mechanics \\ Roza Luxemburg Str. 74, 83 114, Donetsk, Ukraine; aleksdov@mail.ru \\ Mersin University, Faculty of Literature and Science, Department of Mathematics \\ 33342 Mersin, Turkey; mkucukaslan@mersin.edu.tr
}

\begin{abstract}
We find necessary and sufficient conditions for an arbitrary metric space $X$ to have a unique pretangent space at a marked point $a \in X$. Applying this general result we show that each logarithmic spiral has a unique pretangent space at the asymptotic point. Unbounded multiplicative subgroups of $\mathbf{C}^{*}=\mathbf{C} \backslash\{0\}$ having unique pretangent spaces at zero are characterized as lying either on the positive real semiaxis or on logarithmic spirals. Our general uniqueness conditions in the case $X \subseteq \mathbf{R}$ make it also possible to characterize the points of the ternary Cantor set having unique pretangent spaces.
\end{abstract}

\section{Introduction}

Analysis on metric spaces without a smooth structure has recently experienced a rapid development. This development is closely related to some generalizations of the differentiability. Important examples of such generalizations and even an axiomatic approach of so-called "pseudo-gradients" can be found in $[1,3,4,7,15-17,22]$ and in [2]. In almost all aforementioned books and papers the generalized differentiations involve an induced linear structure that makes possible to use the classical differentiations in the linear normed spaces. A new sequential approach to the "smooth" structure for general metric spaces was proposed by Martio and the second author in [14].

A basic technical tool in [14] is the notion of pretangent spaces at a point $a$ of an arbitrary metric space $X$ which were defined as the factor spaces of families of sequences of points $x_{n} \in X$ convergent to $a$. The questions related to the compactness of pretangent spaces were studied in [11]. Certain characterizations of ultrametric pretangent spaces were found in $[12,13]$. The metric differentiation based on the pretangent spaces was introduced in [9]. In the present paper we find necessary and sufficient conditions under which the metric space with a marked point $a$ has a unique pretangent space at $a$ for every normalizing sequence $\tilde{r}$, see Theorem 2.4. Applying these conditions we study the uniqueness of pretangent spaces in the following situations: The point $a$ is a "cusp of the space $X$ " (Section 3); $a$ is the asymptotic point of the logarithmic spiral $X$ (Section 4); for arbitrary $X \subseteq \mathbf{R}$ we characterize the uniqueness conditions in the terms of some cluster sets (Section 5); $a$ is a point of

doi:10.5186/aasfm.2011.3623

2010 Mathematics Subject Classification: Primary 54E35.

Key words: Metric spaces, pretangent spaces, uniqueness of pretangent metric spaces, logarithmic spiral, Cantor set. 
the triadic Cantor set (Section 6). Section 7 contains examples which show that the two conditions for the uniqueness are independent.

For convenience we recall the basic definitions and results from [14], see also [9]. Let $(X, a, d)$ be a pointed metric space with a marked point $a$ and a metric $d$. Fix a sequence $\tilde{r}$ of positive real numbers $r_{n}$ which tend to zero. In what follows this sequence $\tilde{r}$ is called a normalizing sequence. Let us denote by $\tilde{X}$ the set of all sequences of points from $X$.

Definition 1.1. Two sequences $\tilde{x}, \tilde{y} \in \tilde{X}, \tilde{x}=\left\{x_{n}\right\}_{n \in \mathbf{N}}$ and $\tilde{y}=\left\{y_{n}\right\}_{n \in \mathbf{N}}$, are mutually stable (with respect to a normalizing sequence $\tilde{r}=\left\{r_{n}\right\}_{n \in \mathbf{N}}$ ) if there is a finite limit

$$
\lim _{n \rightarrow \infty} \frac{d\left(x_{n}, y_{n}\right)}{r_{n}}:=\tilde{d}_{\tilde{r}}(\tilde{x}, \tilde{y})=\tilde{d}(\tilde{x}, \tilde{y}) .
$$

We shall say that a family $\tilde{F} \subseteq \tilde{X}$ is self-stable (w.r.t. $\tilde{r}$ ) if every two $\tilde{x}, \tilde{y} \in \tilde{F}$ are mutually stable. A family $\tilde{F} \subseteq \tilde{X}$ is maximal self-stable if $\tilde{F}$ is self-stable and for an arbitrary $\tilde{z} \in \tilde{X}$ either $\tilde{z} \in \tilde{F}$ or there is $\tilde{x} \in \tilde{F}$ such that $\tilde{x}$ and $\tilde{z}$ are not mutually stable. A standard application of Zorn's Lemma leads to the following

Proposition 1.2. Let $(X, a, d)$ be a pointed metric space. Then for every normalizing sequence $\tilde{r}=\left\{r_{n}\right\}_{n \in \mathbf{N}}$ there exists a maximal self-stable family $\tilde{X}_{a}=\tilde{X}_{a, \tilde{r}}$ such that $\tilde{a}:=\{a, a, \ldots\} \in \tilde{X}_{a}$.

Note that the condition $\tilde{a} \in \tilde{X}_{a}$ implies the equality

$$
\lim _{n \rightarrow \infty} d\left(x_{n}, a\right)=0
$$

for every $\tilde{x}=\left\{x_{n}\right\}_{n \in \mathbf{N}}$ which belongs to $\tilde{X}_{a}$. Consider a function $\tilde{d}: \tilde{X}_{a} \times \tilde{X}_{a} \rightarrow \mathbf{R}$ where $\tilde{d}(\tilde{x}, \tilde{y})=\tilde{d}_{\tilde{r}}(\tilde{x}, \tilde{y})$ is defined by (1.1). Obviously, $\tilde{d}$ is symmetric and nonnegative. Moreover, the triangle inequality for the original metric $d$ implies

$$
\tilde{d}(\tilde{x}, \tilde{y}) \leq \tilde{d}(\tilde{x}, \tilde{z})+\tilde{d}(\tilde{z}, \tilde{y})
$$

for all $\tilde{x}, \tilde{y}, \tilde{z}$ from $\tilde{X}_{a}$. Hence $\left(\tilde{X}_{a}, \tilde{d}\right)$ is a pseudometric space.

Definition 1.3. The pretangent space to the space $X$ at the point $a$ w.r.t. a normalizing sequence $\tilde{r}$ is the metric identification of the pseudometric space $\left(\tilde{X}_{a, \tilde{r}}, \tilde{d}\right)$.

Since the notion of pretangent space is basic for the present paper, we remind this metric identification construction. Define a relation $\sim$ on $\tilde{X}_{a}$ by $\tilde{x} \sim \tilde{y}$ if and only if $\tilde{d}(\tilde{x}, \tilde{y})=0$. Then $\sim$ is an equivalence relation. Let us denote by $\Omega_{a}=\Omega_{a, \tilde{r}}=\Omega_{a, \tilde{r}}^{X}$ the set of equivalence classes in $\tilde{X}_{a}$ under the equivalence relation $\sim$. It follows from general properties of pseudometric spaces, see, for example, [18, Chapter 4, Th. 15], that if $\rho$ is defined on $\Omega_{a}$ by

$$
\rho_{\tilde{r}}(\alpha, \beta)=\rho(\alpha, \beta):=\tilde{d}(\tilde{x}, \tilde{y})
$$

for $\tilde{x} \in \alpha$ and $\tilde{y} \in \beta$, then $\rho$ is the well-defined metric on $\Omega_{a}$. The metric identification of $\left(\tilde{X}_{a}, \tilde{d}\right)$ is, by definition, the metric space $\left(\Omega_{a}, \rho\right)$.

Remark that $\Omega_{a, \tilde{r}} \neq \emptyset$ because the constant sequence $\tilde{a}$ belongs to $\tilde{X}_{a, \tilde{r}}$, see Proposition 1.2.

Let $\left\{n_{k}\right\}_{k \in \mathbf{N}}$ be an infinite, strictly increasing sequence of natural numbers. Let us denote by $\tilde{r}^{\prime}$ the subsequence $\left\{r_{n_{k}}\right\}_{k \in \mathbf{N}}$ of the normalizing sequence $\tilde{r}=\left\{r_{n}\right\}_{n \in \mathbf{N}}$ 
and let $\tilde{x}^{\prime}:=\left\{x_{n_{k}}\right\}_{k \in \mathbf{N}}$ for every $\tilde{x}=\left\{x_{n}\right\}_{n \in \mathbf{N}} \in \tilde{X}$. It is clear that if $\tilde{x}$ and $\tilde{y}$ are mutually stable w.r.t. $\tilde{r}$, then $\tilde{x}^{\prime}$ and $\tilde{y}^{\prime}$ are mutually stable w.r.t. $\tilde{r}^{\prime}$ and

$$
\tilde{d}_{\tilde{r}}(\tilde{x}, \tilde{y})=\tilde{d}_{\tilde{r}^{\prime}}\left(\tilde{x}^{\prime}, \tilde{y}^{\prime}\right) \text {. }
$$

If $\tilde{X}_{a, \tilde{r}}$ is a maximal self-stable (w.r.t. $\tilde{r}$ ) family, then, by Zorn's Lemma, there exists a maximal self-stable (w.r.t. $\left.\tilde{r}^{\prime}\right)$ family $\tilde{X}_{a, \tilde{r}^{\prime}}$ such that

$$
\left\{\tilde{x}^{\prime}: \tilde{x} \in \tilde{X}_{a, \tilde{r}}\right\} \subseteq \tilde{X}_{a, \tilde{r}^{\prime}} .
$$

Denote by $\operatorname{in}_{\tilde{r}^{\prime}}$ the mapping from $\tilde{X}_{a, \tilde{r}}$ to $\tilde{X}_{a, \tilde{r}^{\prime}}$ with $\operatorname{in}_{\tilde{r}^{\prime}}(\tilde{x})=\tilde{x}^{\prime}$ for all $\tilde{x} \in \tilde{X}_{a, \tilde{r}}$. If follows from (1.2) that after the metric identifications $\mathrm{in}_{\tilde{r}^{\prime}}$ pass to an isometric embedding $\mathrm{em}^{\prime}: \Omega_{a, \tilde{r}} \rightarrow \Omega_{a, \tilde{r}^{\prime}}$ under which the diagram

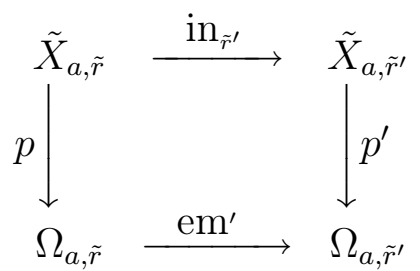

is commutative. Here $p, p^{\prime}$ are the natural projections, $p(\tilde{x}):=\left\{\tilde{y} \in \tilde{X}_{a, \tilde{r}}: \tilde{d}_{\tilde{r}}(\tilde{x}, \tilde{y})=\right.$ $0\}$ and $p^{\prime}(\tilde{x}):=\left\{\tilde{y} \in \tilde{X}_{a, \tilde{r}^{\prime}}: \tilde{d}_{\tilde{r}^{\prime}}(\tilde{x}, \tilde{y})=0\right\}$.

Let $X$ and $Y$ be two metric spaces. Recall that a map $f: X \rightarrow Y$ is called an isometry if $f$ is distance-preserving and onto.

Definition 1.4. A pretangent $\Omega_{a, \tilde{r}}$ is tangent if $\mathrm{em}^{\prime}: \Omega_{a, \tilde{r}} \rightarrow \Omega_{a, \tilde{r}^{\prime}}$ is an isometry for every $\tilde{r}^{\prime}$.

Simple arguments give the following proposition.

Proposition 1.5. Let $(X, a)$ be a pointed metric space, $\tilde{r}$ a normalizing sequence and $\tilde{X}_{a, \tilde{r}}$ a maximal self-stable family with correspondent pretangent space $\Omega_{a, \tilde{r}}$. The following statements are equivalent.

(i) $\Omega_{a, \tilde{r}}$ is tangent.

(ii) For every subsequence $\tilde{r}^{\prime}$ of the sequence $\tilde{r}$ the family $\left\{\tilde{x}^{\prime}: \tilde{x} \in \tilde{X}_{a, \tilde{r}}\right\}$ is $\max$ imal self-stable w.r.t. $\tilde{r}^{\prime}$.

(iii) The function $\mathrm{em}^{\prime}: \Omega_{a, \tilde{r}} \rightarrow \Omega_{a, \tilde{r}^{\prime}}$ is surjective for every $\tilde{r}^{\prime}$.

(iv) The function $\operatorname{in}_{r}^{\prime}: \tilde{X}_{a, \tilde{r}} \rightarrow \tilde{X}_{a, \tilde{r}^{\prime}}$ is surjective for every $\tilde{r}^{\prime}$.

For the proof see [9, Proposition 1.2] or [10, Proposition 1.5].

\section{Conditions of uniqueness of pretangent spaces}

In this section we start from the simplest example of a metric space with unique pretangent spaces.

Example 2.1. Let $X=\mathbf{R}^{+}=[0, \infty[$ be the set of all non-negative, real numbers with the usual metric

$$
d(x, y)=|x-y|
$$

let $\tilde{r}=\left\{r_{n}\right\}_{n \in \mathbf{N}}$ be an arbitrary normalizing sequence and let 0 be the marked point of $X$. Consider a maximal self-stable family $\tilde{X}_{0, \tilde{r}}$.

Proposition 2.2. The following statements are true. 
(i) Let $\tilde{x}=\left\{x_{n}\right\}_{n \in \mathbf{N}} \in \tilde{X}$. Then $\tilde{x} \in \tilde{X}_{0, \tilde{r}}$ if and only if there is $c \geq 0$ such that

$$
\lim _{n \rightarrow \infty} \frac{x_{n}}{r_{n}}=c .
$$

(ii) For every two $\tilde{x}=\left\{x_{n}\right\}_{n \in \mathbf{N}}$ and $\tilde{y}=\left\{y_{n}\right\}_{n \in \mathbf{N}}$ from $\tilde{X}_{0, \tilde{r}}$ the equality

$$
\tilde{d}_{\tilde{r}}(\tilde{x}, \tilde{y})=0
$$

holds if and only if

$$
\lim _{n \rightarrow \infty} \frac{x_{n}}{r_{n}}=\lim _{n \rightarrow \infty} \frac{y_{n}}{r_{n}} .
$$

(iii) The pretangent space $\Omega_{0, \tilde{r}}^{X}$ is isometric to $\left(\mathbf{R}^{+},|\cdot, \cdot|\right)$.

(iv) The pretangent space $\Omega_{0, \tilde{r}}^{X}$ is tangent.

Proof. (i) If $\tilde{x}=\left\{x_{n}\right\}_{n \in \mathbf{N}} \in \tilde{X}_{0, \tilde{r}}$, then there is a finite limit

$$
\lim _{n \rightarrow \infty} \frac{\left|x_{n}-0\right|}{r_{n}}=\tilde{d}(\tilde{x}, \tilde{0}) .
$$

Since we have $x_{n}=\left|x_{n}-0\right|$ for all $n \in \mathbf{N}$, limit relation (2.1) holds with $c=\tilde{d}(\tilde{x}, \tilde{0})$. Suppose that $\tilde{x}, \tilde{y} \in \tilde{X}, \tilde{x}=\left\{x_{n}\right\}_{n \in \mathbf{N}}, \tilde{y}=\left\{y_{n}\right\}_{n \in \mathbf{N}}$ and there are $c_{1}, c_{2} \in \mathbf{R}^{+}$such that

Consequently,

$$
\lim _{n \rightarrow \infty} \frac{x_{n}}{r_{n}}=c_{1}, \lim _{n \rightarrow \infty} \frac{y_{n}}{r_{n}}=c_{2}
$$

$$
\lim _{n \rightarrow \infty} \frac{\left|x_{n}-y_{n}\right|}{r_{n}}=\left|c_{1}-c_{2}\right|,
$$

so that $\tilde{x}$ and $\tilde{y}$ are mutually stable. It implies statement (i).

(ii) Statement (ii) follows from statement (i) and (2.2).

(iii) Define a function $f: \Omega_{0, \tilde{r}}^{X} \rightarrow \mathbf{R}^{+}$by the rule: If $\beta \in \Omega_{0, \tilde{r}}$ and $\tilde{x} \in \beta$, then write $f(\beta):=\lim _{n \rightarrow \infty} \frac{x_{n}}{r_{n}}$. Statements (i),(ii) and limit relation (2.2) imply that $f$ is a well-defined isometry.

(iv) Let $\tilde{n}=\left\{n_{k}\right\}_{k \in \mathbf{N}}$ be a strictly increasing, infinite sequence of natural numbers and let $\tilde{r}^{\prime}=\left\{r_{n_{k}}\right\}_{k \in \mathbf{N}}$ be the corresponding subsequence of the normalizing sequence $\tilde{r}$. If $\tilde{x}=\left\{x_{k}\right\}_{k \in \mathbf{N}} \in \tilde{X}_{0, \tilde{r}^{\prime}}$ then, by statement (i), there is $b \in \mathbf{R}^{+}$such that

$$
\lim _{k \rightarrow \infty} \frac{x_{k}}{r_{n_{k}}}=b .
$$

Define $\tilde{y}=\left\{y_{n}\right\}_{n \in \mathbf{N}} \in \tilde{X}$ as

$$
y_{n}:= \begin{cases}x_{k} & \text { if there is an element } n_{k} \text { of the sequence } \tilde{n} \text { such that } n_{k}=n, \\ b r_{n} & \text { otherwise. }\end{cases}
$$

It is clear that $\tilde{y}^{\prime}=\left\{y_{n_{k}}\right\}_{k \in \mathbf{N}}=\tilde{x}$ and

$$
\lim _{n \rightarrow \infty} \frac{y_{n}}{r_{n}}=b
$$

Hence, by statement (i), $\tilde{y}$ belongs to $\tilde{X}_{0, \tilde{r}}$. Using statement (iv) of Proposition 1.5 we see that $\Omega_{0, \tilde{r}}$ is tangent.

Statement (i) of Proposition 2.2 shows that the pointed space $\left(\mathbf{R}^{+}, 0,|\cdot, \cdot|\right)$ possesses an interesting property: For every normalizing sequence $\tilde{r}$ there exists a unique 
pretangent space $\Omega_{0, \tilde{r}}$. We shall denote by $\mathfrak{U}$ the class of all pointed metric spaces having this property, i.e.

$$
((X, a, d) \in \mathfrak{U}) \Leftrightarrow\left(\forall \tilde{r} \text { there is a unique } \Omega_{a, \tilde{r}}^{X}\right) .
$$

The main results of this paper describe metric spaces belonging to $\mathfrak{U}$.

Remark 2.3. The uniqueness in the previous paragraph and in all theorems below is understood in the usual set-theoretical sense. Statement (i) of Proposition 2.2 implies that for $X=\mathbf{R}^{+}$the family (= the set) $\tilde{X}_{a, \tilde{r}}$ is unique. Hence $\Omega_{0, \tilde{r}}^{X}$, the metric identification of $\tilde{X}_{0, \tilde{r}}$, is also unique. Since the set $\tilde{X}_{0, \tilde{r}}$ is the union of all equivalence classes $\beta \in \Omega_{0, \tilde{r}}$, the uniqueness of the pretangent spaces $\Omega_{0, \tilde{r}}^{X}$ gives the uniqueness of $\tilde{X}_{0, \tilde{r}}$.

Let $(X, a, d)$ be a marked metric space. For each pair of nonvoid sets $C, D \subseteq X$ write

$$
\Delta(C, D):=\sup \{d(x, y): x \in C, y \in D\}
$$

and write

$$
A_{a}(r, k):=\left\{x \in X: \frac{r}{k} \leq d(x, a) \leq r k\right\}, \quad S_{a}(r):=\{x \in X: d(x, a)=r\}
$$

for every $r>0$ and every $k \geq 1$ and define

$$
R_{a, X}:=\left\{r \in \mathbf{R}^{+}: S_{a}(r) \neq \emptyset\right\}
$$

and, for every $\varepsilon \in] 0,1[$

$$
R_{\varepsilon}^{2}:=\left\{(r, t) \in R_{a, X}^{2}: r \neq 0 \neq t \text { and }\left|\frac{r}{t}-1\right| \geq \varepsilon\right\}
$$

where $R_{a, X}^{2}$ is the Cartesian product of $R_{a, X}$ 's. See Figure 1.
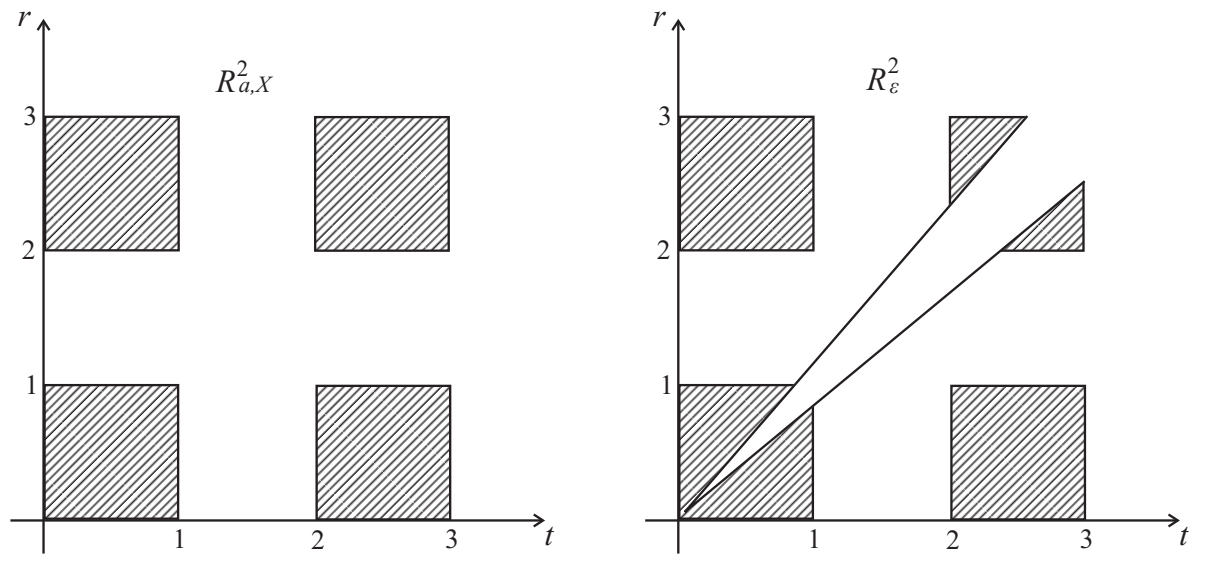

Figure 1. The sets $R_{a, X}^{2}$ and $R_{\varepsilon}^{2}$ with $R_{a, X}=[0,1] \cup[2,3]$ and $\varepsilon=\frac{1}{6}$. Nontengential limit (2.8) is taken over the set $R_{\varepsilon}^{2}$.

Theorem 2.4. Let $(X, a, d)$ be a pointed metric space and let a be a limit point of $X$. Then $(X, a, d) \in \mathfrak{U}$ if and only if the following conditions are satisfied simultaneously.

(i) The limit relation

$$
\left.\lim _{k \rightarrow 1^{+}} \limsup _{r \rightarrow 0^{+}} \frac{\operatorname{diam}\left(A_{a}(r, k)\right)}{r}=0, \quad r \in\right] 0, \infty[, k \in[1, \infty[
$$


holds.

(ii) If $\left\{\left(q_{n}, t_{n}\right)\right\}_{n \in \mathbf{N}}$ is a sequence such that $\left(q_{n}, t_{n}\right) \in R_{\varepsilon}^{2}$ for all $n \in \mathbf{N}$ and

$$
\lim _{n \rightarrow \infty}\left(q_{n}, t_{n}\right)=(0,0)
$$

and there is

$$
\lim _{n \rightarrow \infty} \frac{q_{n}}{t_{n}}=c_{0} \in[0, \infty]
$$

then there exists a finite limit

$$
\lim _{n \rightarrow \infty} \frac{\Delta\left(S_{a}\left(q_{n}\right), S_{a}\left(t_{n}\right)\right)}{\left|q_{n}-t_{n}\right|}:=\varkappa_{0} .
$$

Remark 2.5. The annulus $A_{a}(r, k)$ can be void in (2.4). At that time we use the convention

$$
\operatorname{diam} A_{a}(r, k)=\operatorname{diam}(\emptyset)=0 .
$$

Remark 2.6. If $a$ is an isolated point of $X$ then, obviously, $(X, a, d) \in \mathfrak{U}$ and conditions (i), (ii) of the above theorem is vacuously true.

We need the following lemma.

Lemma 2.7. Let $(X, a, d)$ be a pointed metric space. Then $(X, a, d) \in \mathfrak{U}$ if and only if the implication

$$
\begin{aligned}
& ((\tilde{x} \text { and } \tilde{a} \text { are mutually stable }) \text { and }(\tilde{y} \text { and } \tilde{a} \text { are mutually stable })) \\
& \Longrightarrow(\tilde{x} \text { and } \tilde{y} \text { are mutually stable })
\end{aligned}
$$

is true for every $\tilde{x}, \tilde{y} \in \tilde{X}$.

Proof. Suppose that (2.7) is true. Let $\tilde{X}_{a, \tilde{r}}^{m}$ be the set of all $\tilde{x} \in \tilde{X}$ which are mutually stable with $\tilde{a}$. It follows from (2.7) that $\tilde{X}_{a, \tilde{r}}^{m}$ is self-stable. Consider an arbitrary maximal self-stable $\tilde{X}_{a, \tilde{r}}$, then, by definition of $\tilde{X}_{a, \tilde{r}}$, we obtain the inclusion $\tilde{X}_{a, \tilde{r}}^{m} \supseteq \tilde{X}_{a, \tilde{r}}$. Since $\tilde{X}_{a, \tilde{r}}$ is maximal self-stable, we have also $\tilde{X}_{a, \tilde{r}} \supseteq \tilde{X}_{a, \tilde{r}}^{m}$. Hence the equality

$$
\tilde{X}_{a, \tilde{r}}=\tilde{X}_{a, \tilde{r}}^{m}
$$

holds for all $\tilde{X}_{a, \tilde{r}}$, so all $\tilde{X}_{a, \tilde{r}}$ coincide.

Now suppose that $\tilde{X}_{a, \tilde{r}}$ is unique for every $\tilde{r}$ and there are $\tilde{x}, \tilde{y} \in \tilde{X}$ and there is a normalizing sequence $\tilde{t}$ such that: $\tilde{x}$ and $\tilde{a}$ are mutually stable; $\tilde{y}$ and $\tilde{a}$ are mutually stable; $\tilde{x}$ and $\tilde{y}$ are not mutually stable. By Zorn's Lemma there exist maximal self-stable families $\tilde{X}_{a, \tilde{t}}^{(1)} \supseteq\{\tilde{x}, \tilde{a}\}$ and $\tilde{X}_{a, \tilde{t}}^{(2)} \supseteq\{\tilde{y}, \tilde{a}\}$. It is clear that $\tilde{X}_{a, \tilde{t}}^{(1)} \neq$ $\tilde{X}_{a, \tilde{t}}^{(2)}$. Hence, the uniqueness of pretangent spaces, see Remark 2.3, implies (2.7).

Lemma 2.8. Let $(X, a, d)$ be a pointed metric space and let a be a limit point of $X$. Write

$$
\delta\left(S_{a}(q), S_{a}(t)\right):=\inf \left\{d(x, y): x \in S_{a}(q), y \in S_{a}(t)\right\}
$$

for each $q, t \in R_{a, X}$. If condition (i) of Theorem 2.4 holds, then we have the equality

$$
\lim _{\substack{(t, q) \rightarrow(0,0) \\(t, q) \in R_{\varepsilon}^{2}}} \frac{\Delta\left(S_{a}(q), S_{a}(t)\right)}{\delta\left(S_{a}(q), S_{a}(t)\right)}=1
$$

for every $\varepsilon \in] 0,1[$. 
Proof. Suppose that there is $\varepsilon>0$ such that (2.8) does not hold. Then there exist two sequences of spheres $\left\{S_{a}\left(q_{n}\right)\right\}_{n \in \mathbf{N}},\left\{S_{a}\left(t_{n}\right)\right\}_{n \in \mathbf{N}},\left(t_{n}, q_{n}\right) \in R_{\varepsilon}^{2}$ for all $n \in \mathbf{N}$, and four sequences of points $\left\{x_{n}\right\}_{n \in \mathbf{N}},\left\{y_{n}\right\}_{n \in \mathbf{N}},\left\{z_{n}\right\}_{n \in \mathbf{N}},\left\{p_{n}\right\}_{n \in \mathbf{N}}$ such that

$$
x_{n}, z_{n} \in S_{a}\left(t_{n}\right) \text { and } y_{n}, p_{n} \in S_{a}\left(q_{n}\right)
$$

for all $n \in \mathbf{N}$ and

$$
\begin{aligned}
\lim _{n \rightarrow \infty} x_{n}= & \lim _{n \rightarrow \infty} y_{n}=\lim _{n \rightarrow \infty} z_{n}=\lim _{n \rightarrow \infty} p_{n}=a, \\
& \limsup _{n \rightarrow \infty} \frac{d\left(x_{n}, y_{n}\right)}{d\left(z_{n}, p_{n}\right)}>1 .
\end{aligned}
$$

Suppose also that condition (i) of Theorem 2.4 holds. Then using relation (2.4) with $k=1$ we obtain

$$
\lim _{n \rightarrow \infty} \frac{d\left(x_{n}, z_{n}\right)}{t_{n}}=\lim _{n \rightarrow \infty} \frac{d\left(y_{n}, p_{n}\right)}{q_{n}}=0 .
$$

Note also that the relation $\left(t_{n}, q_{n}\right) \in R_{\varepsilon}^{2}$ and definition (2.3) imply that there is $\left.\left.\varepsilon_{1} \in\right] 0, \varepsilon\right]$ such that the inequalities

$$
\left|q_{n}-t_{n}\right| \geqslant \varepsilon_{1} q_{n} \text { and }\left|t_{n}-q_{n}\right| \geqslant \varepsilon_{1} t_{n}
$$

hold for all $n \in \mathbf{N}$. Let us find the upper bound of the quantity in the left side of (2.4). Write

$$
\eta_{n}:=\frac{d\left(x_{n}, z_{n}\right)}{t_{n}} \text { and } \xi_{n}:=\frac{d\left(y_{n}, p_{n}\right)}{q_{n}}
$$

for all $n \in \mathbf{N}$. The triangle inequality implies

$$
\frac{d\left(x_{n}, y_{n}\right)}{d\left(z_{n}, p_{n}\right)} \leqslant \frac{d\left(x_{n}, z_{n}\right)}{d\left(z_{n}, p_{n}\right)}+\frac{d\left(z_{n}, p_{n}\right)}{d\left(z_{n}, p_{n}\right)}+\frac{d\left(p_{n}, y_{n}\right)}{d\left(z_{n}, p_{n}\right)} \leqslant \eta_{n} \frac{t_{n}}{d\left(z_{n}, p_{n}\right)}+1+\xi_{n} \frac{q_{n}}{d\left(z_{n}, p_{n}\right)} .
$$

Using

$$
d\left(z_{n}, p_{n}\right) \geqslant\left|d\left(z_{n}, a\right)-d\left(p_{n}, a\right)\right|=\left|t_{n}-q_{n}\right|
$$

and (2.11) we obtain

$$
\frac{t_{n}}{d\left(z_{n}, p_{n}\right)} \leqslant \frac{t_{n}}{\left|t_{n}-q_{n}\right|} \leqslant \frac{1}{\varepsilon_{1}} \text { and } \quad \frac{q_{n}}{d\left(z_{n}, p_{n}\right)} \leqslant \frac{q_{n}}{\left|t_{n}-q_{n}\right|} \leqslant \frac{1}{\varepsilon_{1}} .
$$

Thus

$$
\limsup _{n \rightarrow \infty} \frac{d\left(x_{n}, y_{n}\right)}{d\left(z_{n}, p_{n}\right)} \leqslant 1+\frac{1}{\varepsilon_{1}} \limsup _{n \rightarrow \infty}\left(\eta_{n}+\xi_{n}\right) .
$$

The upper limit in the right is zero by (2.10). Consequently

$$
\limsup _{n \rightarrow \infty} \frac{d\left(x_{n}, y_{n}\right)}{d\left(z_{n}, p_{n}\right)} \leqslant 1
$$

contrary to (2.9).

Proof of Theorem 2.4. Assume $(X, a, d) \in \mathfrak{U}$. We need to verify conditions (i)-(ii).

(i) Consider the following function $f:\left[1, \infty\left[\rightarrow \mathbf{R}^{+}\right.\right.$,

$$
f(k):=k \limsup _{r \rightarrow 0} \frac{\operatorname{diam}\left(A_{a}(r, k)\right)}{r} .
$$


Since

$$
f(k):=\limsup _{r \rightarrow 0} \frac{\operatorname{diam}\left(A_{a}\left(k \frac{r}{k}, k\right)\right)}{\frac{r}{k}}=\limsup _{t \rightarrow 0} \frac{\operatorname{diam}\left(A_{a}(k t, k)\right)}{t}
$$

and

$$
A_{a}(k t, k)=\left\{x \in X: t \leq d(x, a) \leq k^{2} t\right\},
$$

the function $f$ is increasing. Since we have

$$
\frac{\operatorname{diam}\left(A_{a}(r, k)\right)}{r} \leq \frac{2 r k}{r}=2 k
$$

for every $k \geq 1$ and all $r>0$, the double inequality

$$
0 \leq f(k) \leq 2 k^{2}
$$

holds. Consequently, there is a finite, positive limit $\lim _{k \rightarrow 1} f(k):=c_{0}$. It is clear that this limit coincides with the limit in (2.4). Suppose that $c_{0}>0$. Let $\left.\varepsilon \in\right] 0, c_{0}[$. Then there is $k_{0}>1$ such that the double inequality

$$
c_{0}-\varepsilon<\limsup _{r \rightarrow 0} \frac{\operatorname{diam}\left(A_{a}(r, k)\right)}{r}<c_{0}+\varepsilon
$$

holds for all $\left.k \in] 1, k_{0}\right]$. Let $\left\{k_{n}\right\}_{n \in \mathbf{N}}$ be a strictly decreasing sequence of real numbers such that all $\left.\left.k_{n} \in\right] 1, k_{0}\right]$ and

$$
\lim _{n \rightarrow \infty} k_{n}=1 \text {. }
$$

Double inequality (2.12) implies that there is a sequence $\tilde{r}=\left\{r_{n}\right\}_{n \in \mathbf{N}}, r_{n}=r_{n}\left(k_{n}\right)>$ 0 , such that $\lim _{n \rightarrow 0} r_{n}=0$ and

$$
c_{0}-\varepsilon<\frac{\operatorname{diam}\left(A_{a}\left(r_{n}, k_{n}\right)\right)}{r_{n}}<c_{0}+\varepsilon
$$

for all $n \in \mathbf{N}$. It follows from (2.14) that there are $\tilde{x}=\left\{x_{n}\right\}_{n \in \mathbf{N}}$ and $\tilde{y}=\left\{y_{n}\right\}_{n \in \mathbf{N}}$ from $\tilde{X}$ such that

$$
x_{n}, y_{n} \in A_{a}\left(r_{n}, k_{n}\right) \quad \text { and } \quad \frac{d\left(x_{n}, y_{n}\right)}{r_{n}} \geq c_{0}-\varepsilon
$$

for all $n \in \mathbf{N}$. The definition of the annulus $A_{a}\left(r_{n}, k_{n}\right)$ and (2.15) imply that

$$
\frac{d\left(x_{n}, a\right)}{r_{n}}, \frac{d\left(y_{n}, a\right)}{r_{n}} \in\left[\frac{1}{k_{n}}, k_{n}\right]
$$

for all $n \in \mathbf{N}$. Define a sequence $\tilde{z}=\left\{z_{n}\right\}_{n \in \mathbf{N}} \in \tilde{X}$ by the rule

$$
z_{n}:= \begin{cases}x_{n} & \text { if } n \text { is even, } \\ y_{n} & \text { if } n \text { is odd }\end{cases}
$$

Then it follows from (2.13), (2.16) and (2.17) that

$$
\lim _{n \rightarrow \infty} \frac{d\left(x_{n}, a\right)}{r_{n}}=\lim _{n \rightarrow \infty} \frac{d\left(z_{n}, a\right)}{r_{n}}=1 .
$$

Moreover, (2.14) and (2.16) imply that

$$
\liminf _{n \rightarrow \infty} \frac{d\left(x_{n}, z_{n}\right)}{r_{n}}=\lim _{n \rightarrow \infty} \frac{d\left(x_{2 n}, z_{2 n}\right)}{r_{2 n}}=0,
$$


but

$$
\limsup _{n \rightarrow \infty} \frac{d\left(x_{n}, z_{n}\right)}{r_{n}}=\limsup _{n \rightarrow \infty} \frac{d\left(x_{2 n+1}, z_{2 n+1}\right)}{r_{2 n+1}} \geq c_{0}-\varepsilon>0 .
$$

Thus $\tilde{x}$ and $\tilde{a}$ are mutually stable, $\tilde{z}$ and $\tilde{a}$ are mutually stable but $\tilde{x}$ and $\tilde{z}$ are not mutually stable (w.r.t. the normalizing sequence $\tilde{r}=\left\{r_{n}\right\}_{n \in \mathbf{N}}$ ). Hence, by Lemma $2.7,(X, a, d) \notin \mathfrak{U}$ contrary to the assumption.

(ii) Let $\left\{\left(q_{n}, t_{n}\right)\right\}_{n \in \mathbf{N}}$ be a sequences of elements of $R_{\varepsilon}^{2}$ such that $\lim _{n \rightarrow \infty}\left(q_{n}, t_{n}\right)$ $=0$ and (2.5) holds. If in (2.5) $c_{0}=0$ or $c_{0}=\infty$, then it is clear that (2.6) holds with $\varkappa_{0}=1$, so it is sufficient to take

$$
0<c_{0}<\infty
$$

Consider the sequence $\tilde{q}=\left\{q_{n}\right\}_{n \in \mathbf{N}}$ as a normalizing sequence. Let $\tilde{x}=\left\{x_{n}\right\}_{n \in \mathbf{N}}$ and $\tilde{y}=\left\{y_{n}\right\}_{n \in \mathbf{N}}$ belong to $\tilde{X}$ and $d\left(a, x_{n}\right)=q_{n}, d\left(a, y_{n}\right)=t_{n}$ and

$$
\lim _{n \rightarrow \infty} \frac{d\left(x_{n}, y_{n}\right)}{\Delta\left(S_{a}\left(q_{n}\right), S_{a}\left(t_{n}\right)\right)}=1 \text {. }
$$

Conditions (2.5) and (2.18) imply that there is

Hence, by Lemma 2.7, there is a finite limit

$$
\tilde{d}_{\tilde{q}}(\tilde{y}, \tilde{a})=\lim _{n \rightarrow \infty} \frac{d\left(y_{n}, a\right)}{q_{n}}=\frac{1}{c_{0}}<\infty .
$$

$$
\tilde{d}_{\tilde{q}}(\tilde{x}, \tilde{y})=\lim _{n \rightarrow \infty} \frac{d\left(x_{n}, y_{n}\right)}{q_{n}} .
$$

Moreover, since $\left(q_{n}, t_{n}\right) \in R_{\varepsilon}^{2}$ for all $n \in \mathbf{N}$, we have $c_{0} \neq 1$. Consequently, using (2.19) and (2.5) we obtain

$$
\begin{aligned}
\lim _{n \rightarrow \infty} \frac{\Delta\left(S_{a}\left(q_{n}\right), S_{a}\left(t_{n}\right)\right)}{\left|q_{n}-t_{n}\right|} & =\lim _{n \rightarrow \infty} \frac{d\left(x_{n}, y_{n}\right) \Delta\left(S_{a}\left(q_{n}\right), S_{a}\left(t_{n}\right)\right)}{q_{n}\left|1-\frac{t_{n}}{q_{n}}\right| d\left(x_{n}, y_{n}\right)} \\
& =\lim _{n \rightarrow \infty} \frac{d\left(x_{n}, y_{n}\right)}{q_{n}} \lim _{n \rightarrow \infty} \frac{1}{\left|1-\frac{t_{n}}{q_{n}}\right|}=\frac{c_{0}}{\left|1-c_{0}\right|} \tilde{d}_{\tilde{q}}(\tilde{x}, \tilde{y}) .
\end{aligned}
$$

Suppose that conditions (i)-(ii) are satisfied simultaneously. We must prove that $\Omega_{a, \tilde{r}}$ is unique for every normalizing sequence $\tilde{r}$. Let $\tilde{r}=\left\{r_{n}\right\}_{n \in \mathbf{N}}$ be an arbitrary normalizing sequence and let $\tilde{x}=\left\{x_{n}\right\}_{n \in \mathbf{N}}$ and $\tilde{y}=\left\{y_{n}\right\}_{n \in \mathbf{N}}$ be two elements of $\tilde{X}$ such that

and

$$
0 \leq \tilde{d}(\tilde{a}, \tilde{x})=\lim _{n \rightarrow \infty} \frac{d\left(a, x_{n}\right)}{r_{n}}<\infty
$$

$$
0 \leq \tilde{d}(\tilde{a}, \tilde{y})=\lim _{n \rightarrow \infty} \frac{d\left(a, y_{n}\right)}{r_{n}}<\infty .
$$

To prove the uniqueness of $\Omega_{a, \tilde{r}}$ it is sufficient, by Lemma 2.7, to show that $\tilde{x}$ and $\tilde{y}$ are mutually stable w.r.t. $\tilde{r}$. If $\tilde{d}(\tilde{a}, \tilde{x})=0$, then, by the triangle inequality,

$$
\limsup _{n \rightarrow \infty} \frac{d\left(x_{n}, y_{n}\right)}{r_{n}} \leq \lim _{n \rightarrow \infty}\left(\frac{d\left(x_{n}, a\right)}{r_{n}}+\frac{d\left(y_{n}, a\right)}{r_{n}}\right)=\tilde{d}(\tilde{a}, \tilde{y})
$$

and

$$
\liminf _{n \rightarrow \infty} \frac{d\left(x_{n}, y_{n}\right)}{r_{n}} \geq \lim _{n \rightarrow \infty}\left(\frac{d\left(y_{n}, a\right)}{r_{n}}-\frac{d\left(x_{n}, a\right)}{r_{n}}\right)=\tilde{d}(\tilde{a}, \tilde{y}) .
$$


Consequently, there is a finite limit

$$
\tilde{d}(\tilde{x}, \tilde{y})=\lim _{n \rightarrow \infty} \frac{d\left(x_{n}, y_{n}\right)}{r_{n}}=d(\tilde{a}, \tilde{y}),
$$

i.e., $\tilde{x}$ and $\tilde{y}$ are mutually stable. The case where $d(\tilde{a}, \tilde{y})=0$ is similar. Hence, without loss of generality we may assume that

$$
\tilde{d}(\tilde{a}, \tilde{y}) \neq 0 \neq d(\tilde{a}, \tilde{x}) .
$$

Consider first the case where

$$
\tilde{d}(\tilde{a}, \tilde{y})=d(\tilde{a}, \tilde{x}):=b \neq 0 .
$$

This assumption implies that for every $k>1$ there is $n_{0}=n_{0}(k) \in \mathbf{N}$ such that the inclusion

$$
A_{a}\left(b r_{n}, k\right) \supseteq\left\{x_{n}, y_{n}\right\}
$$

holds for all natural $n>n_{0}(k)$, where

$$
A_{a}\left(b r_{n}, k\right)=\left\{x \in X: \frac{b r_{n}}{k} \leq d(x, a) \leq k b r_{n}\right\} .
$$

It follows from (2.21) that

$$
d\left(x_{n}, y_{n}\right) \leq \operatorname{diam}\left(A_{a}\left(b r_{n}, k\right)\right)
$$

if $n>n_{0}(k)$. Consequently,

$$
\frac{1}{b} \limsup _{n \rightarrow \infty} \frac{d\left(x_{n}, y_{n}\right)}{r_{n}} \leq \limsup _{n \rightarrow \infty} \frac{\operatorname{diam}\left(A_{a}\left(b r_{n}, k\right)\right)}{b r_{n}} .
$$

Letting $k \rightarrow 1$ on the right-hand side of the last inequality and using (2.4) we see that

Hence

$$
0 \leq \frac{1}{b} \limsup _{n \rightarrow \infty} \frac{d\left(x_{n}, y_{n}\right)}{r_{n}} \leq \lim _{k \rightarrow \infty}\left(\limsup _{n \rightarrow \infty} \frac{\operatorname{diam}\left(A_{a}\left(b r_{n}, k\right)\right)}{b r_{n}}\right)=0 .
$$

It implies that $\tilde{x}$ and $\tilde{y}$ are mutually stable. It still remains to show that there exists a finite limit

if

$$
\tilde{d}(\tilde{x}, \tilde{y})=\lim _{n \rightarrow \infty} \frac{d\left(x_{n}, y_{n}\right)}{r_{n}}
$$

$$
0 \neq \tilde{d}(\tilde{x}, \tilde{a}) \neq \tilde{d}(\tilde{y}, \tilde{a}) \neq 0
$$

For convenience we write

$$
q_{n}:=d\left(x_{n}, a\right), t_{n}:=d\left(y_{n}, a\right)
$$

for all $n \in \mathbf{N}$. Condition (2.23) implies that there are $\varepsilon>0$ and a natural number $n_{0}=n_{0}(\varepsilon)$ such that

$$
q_{n} \wedge t_{n}>0 \text { and } \quad\left|\frac{q_{n}}{t_{n}}-1\right| \geq \varepsilon
$$

for all $n \geq n_{0}$. It is clear that

$$
x_{n} \in S_{a}\left(q_{n}\right) \text { and } y_{n} \in S_{a}\left(t_{n}\right),
$$


where $S_{a}\left(q_{n}\right)$ and $S_{a}\left(t_{n}\right)$ are the spheres with the common center $a \in X$ and radii $q_{n}, t_{n}$, respectively. Consequently, we have the following inequalities

$$
\Delta\left(S_{a}\left(q_{n}\right), S_{a}\left(t_{n}\right)\right) \geq d\left(x_{n}, y_{n}\right) \geq \delta\left(S_{a}\left(q_{n}\right), S_{a}\left(t_{n}\right)\right),
$$

where the quantity $\delta\left(S_{a}\left(q_{n}\right), S_{a}\left(t_{n}\right)\right)$ is defined in Lemma 2.8. Limit relations (2.8) and (2.6) imply that

$$
\varkappa_{0}=\lim _{n \rightarrow \infty} \frac{\Delta\left(S_{a}\left(q_{n}\right), S_{a}\left(t_{n}\right)\right)}{\left|q_{n}-t_{n}\right|}=\lim _{n \rightarrow \infty} \frac{\delta\left(S_{a}\left(q_{n}\right), S_{a}\left(t_{n}\right)\right)}{\left|q_{n}-t_{n}\right|} .
$$

Hence, using (2.25), we obtain

$$
\varkappa_{0}=\lim _{n \rightarrow \infty} \frac{d\left(x_{n}, y_{n}\right)}{\left|q_{n}-t_{n}\right|}=\frac{1}{|\tilde{d}(\tilde{x}, \tilde{a})-\tilde{d}(\tilde{y}, \tilde{a})|} \lim _{n \rightarrow \infty} \frac{d\left(x_{n}, y_{n}\right)}{r_{n}},
$$

that implies

$$
\tilde{d}(\tilde{x}, \tilde{y})=\lim _{n \rightarrow \infty} \frac{d\left(x_{n}, y_{n}\right)}{r_{n}}=\varkappa_{0}|\tilde{d}(\tilde{x}, \tilde{a})-\tilde{d}(\tilde{y}, \tilde{a})|,
$$

i.e., $\tilde{x}$ and $\tilde{y}$ are mutually stable.

It will be proved in the Section 7 of the paper that conditions (i) and (ii) of Theorem 2.4 are mutually independent in the sense that no one of them implies the another.

The next proposition follows from Lemma 2.7.

Proposition 2.9. Let $(X, a, d)$ be a pointed metric space, let $Y \subseteq X$ and let $a \in Y$. Then the relation $(X, a, d) \in \mathfrak{U}$ implies $(Y, a, d) \in \mathfrak{U}$.

\section{Examples of metric spaces with unique pretangent spaces}

Using Example 2.1 as a model we can construct more geometrically interesting examples of metric spaces with unique tangent spaces. To this end we recall first some facts related to the structure of pretangent spaces to subspaces of metric spaces.

Let $(X, a, d)$ be a pointed metric space, let $Y$ and $Z$ be subspaces of $X$ such that $a \in Y \cap Z$ and let $\tilde{r}=\left\{r_{n}\right\}_{n \in \mathbf{N}}$ be a normalizing sequence.

Definition 3.1. The pointed spaces $(Y, a)$ and $(Z, a), a \in X \cap Y$, are tangent equivalent at $a$ w.r.t. normalizing sequence $\tilde{r}$ if for every $\tilde{y}_{1}=\left\{y_{n}^{(1)}\right\}_{n \in \mathbf{N}} \in \tilde{Y}$ and every $\tilde{z}_{1}=\left\{z_{n}^{(1)}\right\}_{n \in \mathbf{N}} \in \tilde{Z}$ with finite limits

$$
\tilde{d}_{\tilde{r}}\left(\tilde{a}, \tilde{y}_{1}\right)=\lim _{n \rightarrow \infty} \frac{d\left(y_{n}^{(1)}, a\right)}{r_{n}} \quad \text { and } \quad \tilde{d}_{\tilde{r}}\left(\tilde{a}, \tilde{z}_{1}\right)=\lim _{n \rightarrow \infty} \frac{d\left(z_{n}^{(1)}, a\right)}{r_{n}}
$$

there exist $\tilde{y}_{2}=\left\{y_{n}^{(2)}\right\}_{n \in \mathbf{N}} \in \tilde{Y}$ and $\tilde{z}_{2}=\left\{z_{n}^{(2)}\right\}_{n \in \mathbf{N}} \in \tilde{Z}$ such that

$$
\lim _{n \rightarrow \infty} \frac{d\left(y_{n}^{(1)}, z_{n}^{(2)}\right)}{r_{n}}=\lim _{n \rightarrow \infty} \frac{d\left(y_{n}^{(2)}, z_{n}^{(1)}\right)}{r_{n}}=0 .
$$

We shall say that $(Y, a)$ and $(Z, a)$ are strongly tangent equivalent if $(Y, a)$ and $(Z, a)$ are tangent equivalent for all normalizing sequences $\tilde{r}$.

Let $\tilde{F} \subseteq \tilde{X}$. For a normalizing sequence $\tilde{r}$ we define a family $[\tilde{F}]_{Y}=[\tilde{F}]_{Y, \tilde{r}}$ by the rule

$$
\left(\tilde{y} \in[\tilde{F}]_{Y}\right) \Leftrightarrow\left((\tilde{y} \in \tilde{Y}) \text { and }\left(\exists \tilde{x} \in \tilde{F}: \tilde{d}_{\tilde{r}}(\tilde{x}, \tilde{y})=0\right)\right)
$$


The following two lemmas were proved in [9], see also [14].

Lemma 3.2. Let $Y$ and $Z$ be subspaces of a metric space $X, a \in Y \cap Z$ and let $\tilde{r}$ be a normalizing sequence. Suppose that $(Y, a)$ and $(Z, a)$ are tangent equivalent w.r.t. $\tilde{r}$. Then following statements hold for every maximal self-stable (in $\tilde{Z}$ ) family $\tilde{Z}_{a, \tilde{r}}$.

(i) The family $\left[\tilde{Z}_{a, \tilde{r}}\right]_{Y}$ is maximal self-stable (in $\tilde{Y}$ ) and we have the equality

$$
\left[\left[\tilde{Z}_{a, \tilde{r}}\right]_{Y}\right]_{Z}=\tilde{Z}_{a, \tilde{r}}
$$

(ii) If $\Omega_{a, \tilde{r}}^{Z}$ and $\Omega_{a, \tilde{r}}^{Y}$ are metric identifications of $\tilde{Z}_{a, \tilde{r}}$ and, respectively, of $\tilde{Y}_{a, \tilde{r}}:=$ $\left[\tilde{Z}_{a, \tilde{r}}\right]_{Y}$, then the mapping

$$
\Omega_{a, \tilde{r}}^{Z} \ni \alpha \longmapsto[\alpha]_{Y} \in \Omega_{a, \tilde{r}}^{Y}
$$

is an isometry. Furthermore, if $\Omega_{a, \tilde{r}}^{Z}$ is tangent, then $\Omega_{a, \tilde{r}}^{Y}$ also is tangent.

(iii) If for the normalizing sequence $\tilde{r}$ there exists a unique maximal self-stable (in $\tilde{Z}$ ) family $\tilde{Z}_{a, \tilde{r}} \ni \tilde{a}$, then $\tilde{Y}_{a, \tilde{r}}:=\left[\tilde{Z}_{a, \tilde{r}}\right]_{Y}$ is a unique maximal self-stable (in $\tilde{Y}$ ) family which contains $\tilde{a}$.

Let $Y$ be a subspace of a metric space $(X, d)$. For $a \in Y$ and $t>0$ we denote by

$$
S_{t}^{Y}=S^{Y}(a, t):=\{y \in Y: d(a, y)=t\}
$$

the sphere (in the subspace $Y$ ) with the center $a$ and the radius $t$. Similarly for $a \in Z \subseteq X$ and $t>0$ define

$$
S_{t}^{Z}=S^{Z}(a, t):=\{z \in Z: d(a, z)=t\} .
$$

Write

$$
\varepsilon_{a}(t, Z, Y):=\sup _{z \in S_{t}^{Z}} \inf _{y \in Y} d(z, y)
$$

and

$$
\varepsilon_{a}(t):=\varepsilon_{a}(t, Z, Y) \vee \varepsilon_{a}(t, Y, Z) .
$$

Lemma 3.3. Let $Y$ and $Z$ be subspaces of a metric space $(X, d)$ and let $a \in$ $Y \cap Z$. Then $Y$ and $Z$ are strongly tangent equivalent at the point $a$ if and only if the equality

$$
\lim _{t \rightarrow 0} \frac{\varepsilon_{a}(t)}{t}=0
$$

holds.

Remark 3.4. Statement (iii) of Lemma 3.2 implies, in particular, that

$$
((Y, a) \in \mathfrak{U}) \Leftrightarrow((Z, a) \in \mathfrak{U})
$$

if $(Y, a)$ and $(Z, a)$ are strongly tangent equivalent.

Using Proposition 2.2, Lemma 3.2 and Lemma 3.3 we can easily obtain examples of subspaces of Euclidean spaces which have unique tangent spaces in their cusps. The first example will be examined in details.

Example 3.5. Let $F:[0,1] \rightarrow E^{n}, n \geq 2$, be a Jordan curve in the Euclidean space $E^{n}$, i.e., $F$ is continuous and

$$
F\left(t_{1}\right) \neq F\left(t_{2}\right)
$$


for every two distinct points $t_{1}, t_{2} \in[0,1]$. We can write $F$ in the coordinate form

$$
F(t)=\left(f_{1}(t), \ldots, f_{n}(t)\right), \quad t \in[0,1] .
$$

Suppose that all functions $f_{i}, 1 \leq i \leq n$, are differentiable at the point 0 and

$$
F^{\prime}(0)=\left(f_{1}^{\prime}(0), \ldots, f_{n}^{\prime}(0)\right) \neq(0, \ldots, 0) .
$$

(We use the one-sided derivatives here.) We claim that each pretangent space to the space $Y=F([0,1]) \subseteq E^{n}$ at the point $a=F(0)$ is unique and tangent and isometric to $\mathbf{R}^{+}$for every normalizing sequence $\tilde{r}$. Indeed, by Lemma 3.2 and by Proposition 2.2 , it is sufficient to show that $Y$ is strongly tangent equivalent to the ray

$$
Z=\left\{\left(z_{1}(t), \ldots, z_{n}(t)\right):\left(z_{1}(t), \ldots, z_{n}(t)\right)=t F^{\prime}(0)+F(0), t \in \mathbf{R}^{+}\right\}
$$

at the point $a=F(0)$.

The classical definition of the differentiability of real functions shows that limit relation (3.1) holds with these $Y$ and $Z$. Hence, by Lemma 3.3, $Y$ and $Z$ are strongly tangent equivalent at the point $a=F(0)$.

Example 3.6. Let $f_{i}:[0,1] \rightarrow \mathbf{R}, i=1, \ldots, n$, be functions such that $f_{1}(0)=$ $\cdots=f_{n}(0)=c$ where $c \in \mathbf{R}$ is a constant. Suppose all $f_{i}$ have a common finite right derivative $b$ at the point $0, f_{1}^{\prime}(0)=\cdots=f_{n}^{\prime}(0)=b$. Write

$$
a=(0, c) \quad \text { and } \quad X=\bigcup_{i=1}^{n}\left\{\left(t, f_{i}(t)\right): t \in[0,1]\right\},
$$

i.e., $X$ is an union of the graphs of the functions $f_{i}$. Let us consider $X$ as a subspace of the Euclidean plane $E^{2}$. Then for every normalizing sequence $\tilde{r}$ a pretangent space $\tilde{\Omega}_{a, \tilde{r}}$ to the space $X$ at the point $a$ is unique, tangent and isometric to $\mathbf{R}^{+}$.

Example 3.7. Let $f_{1}, f_{2}$ be two functions from the precedent example. Put

$$
X=\left\{(x, y): f_{1}(x) \wedge f_{2}(x) \leq y \leq f_{1}(x) \vee f_{2}(x), x \in[0,1]\right\},
$$

i.e., $X$ is the set of points of the plane which lie between the graphs of the functions $f_{1}$ and $f_{2}$. Then for every normalizing sequence $\tilde{r}$ each pretangent space $\tilde{\Omega}_{a, \tilde{r}}^{X}$ to $X$ at $a=(0, c)$ is unique, tangent and isometric to $\mathbf{R}^{+}$.

Example 3.8. Let $\alpha$ be a positive real number. Write

$$
X=\left\{(x, y, z) \in E^{3}: \sqrt{y^{2}+z^{2}} \leq x^{1+\alpha}, x \in \mathbf{R}^{+}\right\},
$$

i.e., $X$ can be obtained by the rotation of the plane figure

$$
\left\{(x, y) \in E^{2}: 0 \leq y \leq x^{1+\alpha}, x \in \mathbf{R}^{+}\right\}
$$

around the real axis. Then each pretangent space $\tilde{\Omega}_{a, \tilde{r}}$ to $X$ at the point $a=(0,0,0)$ is unique, tangent and isometric to $\mathbf{R}^{+}$.

In all examples above pretangent spaces $\Omega_{a, \tilde{r}}^{X}$ were also tangent. The following example shows that there is a metric space $X$ for which $\Omega_{a, \tilde{r}}^{X}$ is unique but not tangent.

Example 3.9. Let $\tilde{r}=\left\{r_{n}\right\}_{n \in \mathbf{N}}$ be a sequence of strictly decreasing positive real numbers $r_{n}$ with

$$
\lim _{n \rightarrow \infty} \frac{r_{n}}{r_{n+1}}=\infty
$$


and such that $r_{n}>2 r_{n+1}$ for all $n \in \mathbf{N}$. Let $X$ be a union of two countable sets $\left\{r_{n}: n \in \mathbf{N}\right\}$ and $\left\{2 r_{2 n}: n \in \mathbf{N}\right\}$ and the one-point set $\{0\}$,

$$
X=\left\{r_{n}: n \in \mathbf{N}\right\} \cup\left\{2 r_{2 n}: n \in \mathbf{N}\right\} \cup\{0\} .
$$

Consider the metric space $(X,|\cdot, \cdot|)$. It is clear that the sequences $\tilde{0}$ and $\tilde{x}:=\left\{r_{n}\right\}_{n \in \mathbf{N}}$ are mutually stable w.r.t. $\tilde{r}$ and

$$
\tilde{d}_{\tilde{r}}(\tilde{x}, \tilde{0})=1 .
$$

Let $\tilde{X}_{0, \tilde{r}}$ be a unique (by Proposition 2.9) maximal self-stable family such that

$$
\tilde{X}_{0, \tilde{r}} \supseteq\{0, \tilde{x}\} .
$$

We claim that the pretangent space $\Omega_{0, \tilde{r}}^{X}$ corresponding to $\tilde{X}_{0, \tilde{r}}$ is two-point. Indeed, suppose that $\tilde{y}=\left\{y_{n}\right\}_{n \in \mathbf{N}} \in \tilde{X}_{0, \tilde{r}}$ and $\tilde{d}(\tilde{y}, \tilde{0})>0$. It is sufficient to prove the equality

$$
\tilde{d}(\tilde{x}, \tilde{y})=0 .
$$

To this end, we note that (3.2) and (3.3) imply

$$
\frac{y_{2 n+1}}{r_{2 n+1}}=1 \quad \text { and } \quad \frac{y_{2 n}}{r_{2 n}} \in\{1,2\}
$$

for all sufficiently large $n \in \mathbf{N}$ because in the opposite case

$$
\text { either } \lim _{n \rightarrow \infty} \frac{y_{n}}{r_{n}}=0 \quad \text { or } \quad \lim _{n \rightarrow \infty} \frac{y_{n}}{r_{n}}=\infty \text {. }
$$

Since

$$
1=\lim _{n \rightarrow \infty} \frac{y_{2 n+1}}{r_{2 n+1}}=\lim _{n \rightarrow \infty} \frac{y_{n}}{r_{n}}=\lim _{n \rightarrow \infty} \frac{y_{2 n}}{r_{2 n}},
$$

conditions (3.5) imply that

$$
y_{2 n}=r_{2 n}
$$

for sufficiently large $n$. Hence (3.4) follows.

Now let $\tilde{r}^{\prime}:=\left\{r_{2 n}\right\}_{n \in \mathbf{N}}$ and $\tilde{X}_{0, \tilde{r}^{\prime}}$ be a maximal self-stable family such that

$$
\tilde{X}_{0, \tilde{r}^{\prime}} \supseteq\{\tilde{0}, \tilde{x}, \tilde{z}\}
$$

where $\tilde{x}:=\left\{r_{2 n}\right\}_{n \in \mathbf{N}}$ and $\tilde{z}:=\left\{2 r_{2 n}\right\}_{n \in \mathbf{N}}$. Since

$$
1=\tilde{d}_{\tilde{r}^{\prime}}(\tilde{0}, \tilde{x})=\frac{1}{2} \tilde{d}_{\tilde{r}^{\prime}}(\tilde{0}, \tilde{z})=\tilde{d}_{\tilde{r}^{\prime}}(\tilde{x}, \tilde{z}),
$$

the pretangent space $\Omega_{0, \tilde{r}^{\prime}}$ corresponding to $\tilde{X}_{0, \tilde{r}^{\prime}}$ contains at least three distinct points. Consequently, $\Omega_{0, \tilde{r}}$ is not tangent.

Remark 3.10. There are pointed metric spaces $(X, a)$ for which all pretangent spaces $\Omega_{a, \tilde{r}}^{X}$ are tangent but $(X, a) \notin \mathfrak{U}$. As an example we can take $X=\mathbf{C}$ of $X=\mathbf{R}$, see [10].

In the next section of the paper we will describe the tangent space to the logarithmic spiral at its asymptotic point. 


\section{Uniqueness of pretangent spaces and logarithmic spirals}

We will consider only logarithmic spirals having the asymptotic point at 0 . The polar equation of these spirals is

$$
\rho=k b^{\varphi}
$$

where $k$ and $b$ are constants, $k \in] 0, \infty[$ and $b \in] 0,1[\cup] 1, \infty[$. The rotation of the polar axis on the angle $\varphi_{1}=-\frac{\ln k}{\ln b}$ transforms (4.1) to the form

$$
\rho=b^{\varphi} \text {. }
$$

Let us denote by $S^{*}=S^{*}(b)$ the set of all complex numbers lying on spiral (4.2) and let

$$
S=S^{*} \cup\{0\}
$$

i.e., $S$ is the closure of $S^{*}$ in the complex plane C. In the following theorems we consider $S$ as a metric space with the usual metric $d(z, w)=|z-w|$.

Theorem 4.1. Each pretangent space to $S$ at the point 0 is unique, tangent and isometric to $S$.

Denote by $\mathbf{C}^{*}$ the multiplicative group of all nonzero complex numbers. We shall need the following lemma.

Lemma 4.2. $S^{*}$ is a subgroup of the group $\mathbf{C}^{*}$.

Proof. As is well known, a nonvoid subset $H$ of a group $G$ is a subgroup of $G$ if and only if

$$
h g \in H \quad \text { and } \quad h^{-1} \in H
$$

for all $h, g \in H$. See, for example, [19, Chapter $1, \S 2]$. Let $z$ be a point of $\mathbf{C}^{*}$. It is clear from (4.2) that $z \in S^{*}$ if and only if

$$
z=|z| \exp \left(i \log _{b}|z|\right) .
$$

The last equality implies

$$
z^{-1}=|z|^{-1} \exp \left(-i \log _{b}|z|\right)=\left|z^{-1}\right| \exp \left(i \log _{b}\left|z^{-1}\right|\right) .
$$

Hence $z^{-1}$ belongs to $S^{*}$ if $z \in S^{*}$. Similarly we obtain $z w \in S^{*}$ if $z \in S^{*}$ and $w \in S^{*}$.

The next useful lemma describes the isometries of metric identifications of pseudometric spaces.

Lemma 4.3. Let $\left(X, d_{X}\right)$ be a pseudometric space, $\left(Y, d_{Y}\right)$ a metric space, $(\Omega, \rho)$ a metric identification of $\left(X, d_{X}\right)$ and $p: X \rightarrow \Omega$ the natural projection. Then for every distance-preserving, surjective mapping $F: X \rightarrow Y$ there is a unique mapping $f: \Omega \rightarrow Y$ such that the diagram

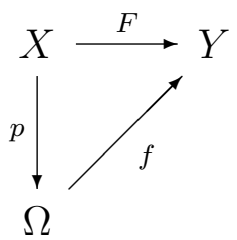

is commutative. This $f$ is an isometry. 
Proof. Let us define a mapping $f$ by the rule: if $\alpha \in \Omega$, then

$$
f(\alpha)=F(x),
$$

where $x$ is an arbitrary point in $p^{-1}(\alpha)$. This definition is correct. Indeed, if $x_{1}$, $x_{2} \in p^{-1}(\alpha)$, then $d_{X}\left(x_{1}, x_{2}\right)=0$ because $p$ is natural projection. The equality $d_{X}\left(x_{1}, x_{2}\right)=0$ implies $d_{Y}\left(F\left(x_{1}\right), F\left(x_{2}\right)\right)=0$ because $F$ is distance-preserving. Since $d_{Y}$ is a metric, the last equality gives $F\left(x_{1}\right)=F\left(x_{2}\right)$.

Rewriting (4.5) as $f(p(x))=F(x)$ we see that the diagram is commutative. The uniqueness of $f$ which satisfies the equality $F=f \circ p$ follows from the surjectivity of $p$. It still remains to prove that $F$ is an isometry.

Let $\alpha, \beta \in \Omega, x, y \in X$ and $\alpha=p(x), \beta=p(y)$. Then we have

$$
\begin{aligned}
d_{Y}(f(\alpha), f(\beta)) & =d_{Y}(f(p(x)), f(p(y)))=d_{Y}(F(x), F(y)) \\
& =d_{X}(x, y)=\rho(p(x), p(y))=\rho(\alpha, \beta) .
\end{aligned}
$$

Thus $f$ is distance-preserving. Moreover $f$ is surjective because $F$ is surjective. Hence $f$ is an isometry as a distance-preserving, surjective mapping between metric spaces.

Proof of Theorem 4.1. We shall first prove $(S, 0) \in \mathfrak{U}$. To this end it is sufficient to show that conditions (i)-(ii) from Theorem 2.4 are satisfied with $X=S$ and $a=0$.

We start from the verification of

Condition (i). Let $z_{1}$ and $z_{2}$ be some points of the annulus

$$
A_{0}(r, k)=\left\{z \in S: \frac{r}{k} \leq|z| \leq k z\right\},
$$

where $k \in] 1, \infty[, r \in] 0, \infty\left[\right.$. Let us denote by $l\left(z_{1}, z_{2}\right)$ the length of the arc of spiral (4.2) joining the points $z_{1}, z_{2}$. If the polar coordinates of $z_{1}$ and $z_{2}$ are $\left(\rho_{1}, \varphi_{1}\right)$ and $\left(\rho_{2}, \varphi_{2}\right)$ respectively, then we obtain the famous formula

$$
l\left(z_{1}, z_{2}\right)=\left|\int_{\varphi_{1}}^{\varphi_{2}} \sqrt{\rho^{2}+\rho^{\prime 2}} d \varphi\right|=\frac{\sqrt{1+\ln ^{2} b}}{|\ln b|}\left|b^{\varphi_{2}}-b^{\varphi_{1}}\right|=\frac{\sqrt{1+\ln ^{2} b}}{|\ln b|}\left|\rho_{2}-\rho_{1}\right| .
$$

It implies that

$$
\begin{aligned}
\operatorname{diam} A_{0}(r, k) & \leq \sup \left\{l\left(z_{1}, z_{2}\right): z_{1}, z_{2} \in A_{0}(r, k)\right\} \\
& =\frac{\sqrt{1+\ln ^{2} b}}{|\ln b|}\left|r k-r \frac{1}{k}\right|=\frac{r \sqrt{1+\ln ^{2} b}}{|\ln b|}\left(k-\frac{1}{k}\right) .
\end{aligned}
$$

Consequently,

$$
\lim _{k \rightarrow 1} \limsup _{r \rightarrow 0} \frac{\operatorname{diam}\left(A_{a}(r, k)\right)}{r} \leq \lim _{k \rightarrow 1} \frac{\sqrt{1+\ln ^{2} b}}{|\ln b|}\left(k-\frac{1}{k}\right)=0,
$$

i.e., (2.4) holds and condition (i) is satisfied.

Condition (ii). Let $\varepsilon \in] 0,1\left[\right.$ and let $\left\{\left(q_{n}, t_{n}\right)\right\}_{n \in \mathbf{N}}$ be a sequence of points of $R_{\varepsilon}^{2}$, see Figure 1, such that

$$
\lim _{n \rightarrow \infty}\left(q_{n}, t_{n}\right)=(0,0)
$$

and there is

$$
\lim _{n \rightarrow \infty} \frac{q_{n}}{t_{n}}=c_{0} \in[0, \infty] .
$$


We must show that there exists a finite limit

$$
\lim _{n \rightarrow \infty} \frac{\Delta\left(S_{0}\left(q_{n}\right), S_{0}\left(t_{n}\right)\right)}{\left|q_{n}-t_{n}\right|}=\varkappa .
$$

It follows from the definition of the set $R_{\varepsilon}^{2}$ that $\left.q_{n}, t_{n} \in\right] 0, \infty[$ for all $n \in \mathbf{N}$. Consequently, we can find $\left.\theta_{n}, \tau_{n} \in\right]-\infty,+\infty[$ such that

$$
q_{n} e^{i \tau_{n}} \in S^{*} \text { and } t_{n} e^{i \theta_{n}} \in S^{*}
$$

for all $n \in \mathbf{N}$. Since the spheres $S_{0}\left(q_{n}\right)$ and $S_{0}\left(t_{n}\right)$ are one-point and $q_{n} e^{i \tau_{n}} \in S_{0}\left(q_{n}\right)$ and $t_{n} e^{i \theta_{n}} \in S_{0}\left(t_{n}\right)$, we have

$$
\frac{\Delta\left(S_{0}\left(t_{n}\right), S_{0}\left(q_{n}\right)\right)}{\left|t_{n}-q_{n}\right|}=\frac{\left|t_{n} e^{i \theta_{n}}-q_{n} e^{i \tau_{n}}\right|}{\left|t_{n}-q_{n}\right|}
$$

with

$$
\tau_{n}=\log _{b} q_{n}, \quad \theta_{n}=\log _{b} t_{n},
$$

see (4.3). Consider firstly the cases where $c_{0}=0$ or $c_{0}=\infty$ in equation (4.8). If $c_{0}=0$, then it implies that

$$
\lim _{n \rightarrow \infty} \frac{\left|t_{n} e^{i \theta_{n}}-q_{n} e^{i \tau_{n}}\right|}{\left|t_{n}-q_{n}\right|}=\lim _{n \rightarrow \infty} \frac{\left|1-\frac{q_{n}}{t_{n}} e^{i\left(\tau_{n}-\theta_{n}\right)}\right|}{\left|1-\frac{q_{n}}{t_{n}}\right|}=\frac{\left|1-c_{0}\right|}{\left|1-c_{0}\right|}=1=\varkappa .
$$

Similar computations yield $\varkappa=1$ for $c_{0}=\infty$. Suppose now $\left.c_{0} \in\right] 0, \infty[$. Using (4.8) and (4.11) we obtain

$$
\lim _{n \rightarrow \infty}\left(\tau_{n}-\theta_{n}\right)=\lim _{n \rightarrow \infty}\left(\log _{b} q_{n}-\log _{b} t_{n}\right)=\lim _{n \rightarrow \infty} \log _{b} \frac{q_{n}}{t_{n}}=\log _{b} c_{0} .
$$

Moreover, we have

$$
c_{0} \neq 1
$$

because $\left(q_{n}, t_{n}\right) \in R_{\varepsilon}^{2}$ for all $n \in \mathbf{N}$. Applying (4.13) in computations (4.12) we obtain

$$
\lim _{n \rightarrow \infty} \frac{\left|t_{n} e^{i \theta_{n}}-q_{n} e^{i \tau_{n}}\right|}{\left|t_{n}-q_{n}\right|}=\frac{1-c_{0} \exp \left(i \log _{b} c_{0}\right)}{\left|1-c_{0}\right|} .
$$

Note that the right side in this equality is finite and correctly defined by virtue of (4.14).

Thus conditions (i)-(ii) from Theorem 2.4 are satisfied, so that this theorem implies the desirable uniqueness of pretangent spaces. It still remains to prove that all pretangent spaces are tangent and isometric to $S$.

Let $\tilde{r}=\left\{r_{n}\right\}_{n \in \mathbf{N}}$ be a normalizing sequence and let $\tilde{S}_{0, \tilde{r}}$ be a corresponding maximal self-stable family. For every $\tilde{x}=\left\{x_{n}\right\}_{n \in \mathbf{N}} \in \tilde{S}_{0, \tilde{r}}$ there is a unique $x^{*} \in S$ such that

$$
\left|x^{*}\right|=\lim _{n \rightarrow \infty} \frac{d\left(x_{n}, 0\right)}{r_{n}}=\tilde{r}(\tilde{x}, \tilde{0}) .
$$

We claim that the mapping

$$
F: \tilde{S}_{0, \tilde{r}} \rightarrow S, \quad F(\tilde{x})=x^{*}
$$

is surjective and distance-preserving in the sense that the equality

$$
\tilde{d}_{\tilde{r}}(\tilde{x}, \tilde{y})=\left|x^{*}-y^{*}\right|
$$

holds for all $\tilde{x}, \tilde{y} \in \tilde{S}_{0, \tilde{r}}$. 
Surjectivity. We have already verified that $(S, 0) \in \mathfrak{U}$. Hence, by Lemma 2.7, it is sufficient to prove that for every $a \in S$ there is $\tilde{x} \in \tilde{S}$ such that $\left|x^{*}\right|=|a|$. If $|a|=0$, then the equality $\left|x^{*}\right|=|a|$ is evident for $\tilde{x}=(0,0, \ldots)$. Assume that $|a|>0$. For every $n \in \mathbf{N}$ define a complex number $r_{n}^{0}$ as

$$
r_{n}^{0}:=\left|\frac{1}{r_{n}}\right| \exp \left(i \log \left|\frac{1}{r_{n}}\right|\right),
$$

see formula (4.3). The points $r_{n}^{0}$ and $x^{*}$ belong to $S^{*}$. By Lemma 4.2 the set $S^{*}$ is a subgroup of $\mathbf{C}^{*}$. Since the equation $a y=b$ is solvable in every group, there is $x_{n} \in S^{*}$ such that

$$
r_{n}^{0} x_{n}=a
$$

This equality and (4.18) imply

$$
\frac{d\left(x_{n}, 0\right)}{r_{n}}=\left|\frac{x_{n}}{r_{n}}\right|=|a|
$$

for all $n \in \mathbf{N}$. Thus the equality $|a|=\left|x^{*}\right|$ holds for $\tilde{x}:=\left\{x_{n}\right\}_{n \in \mathbf{N}}$ if all $x_{n}$ fulfil equations (4.19).

Preservation of distances. Let $\tilde{x}=\left\{x_{n}\right\}_{n \in \mathbf{N}}$ and $\tilde{y}=\left\{y_{n}\right\}_{n \in \mathbf{N}}$ belong to $\tilde{S}_{0, \tilde{r}}$. If $x^{*}=0$ or $y^{*}=0$, then equality (4.17) follows simply from (4.15). Assume that $x^{*} \neq 0 \neq y^{*}$. This assumption implies that $x^{*} \in S^{*}, y^{*} \in S^{*}$ and

$$
x_{n} \in S^{*}, \quad y_{n} \in S^{*}
$$

for all sufficiently large $n \in \mathbf{N}$. These membership relations give, in particular, the equalities

$$
x^{*}=\left|x^{*}\right| \exp \left(i \log _{b}\left|x^{*}\right|\right), \quad x_{n}=\left|x_{n}\right| \exp \left(i \log _{b}\left|x_{n}\right|\right),
$$

see (4.3). Moreover, we can rewrite (4.18) as

$$
r_{n}^{0}=\left|r_{n}^{0}\right| \exp \left(i \log _{b}\left|r_{n}^{0}\right|\right)
$$

Using it, (4.20) and (4.15) we obtain

$$
\lim _{n \rightarrow \infty}\left|r_{n}^{0}\right|\left|x_{n}\right|=\left|x^{*}\right|
$$

Consequently,

$$
\lim _{n \rightarrow \infty} \exp \left(i \log _{b}\left(\left|r_{n}^{0}\right|\left|x_{n}\right|\right)\right)=\exp \left(i \log _{b}\left|x^{*}\right|\right) .
$$

Relations (4.20)-(4.22) give the equality

$$
\lim _{n \rightarrow \infty} r_{n}^{0} x_{n}=x^{*}
$$

Similarly, we have $\lim _{n \rightarrow \infty} r_{n}^{0} y_{n}=y^{*}$. The last two equality imply

$$
\left|x^{*}-y^{*}\right|=\left|\lim _{n \rightarrow \infty}\left(r_{n}^{0} x_{n}-r_{n}^{0} y_{n}\right)\right|=\lim _{n \rightarrow \infty} \frac{\left|x_{n}-y_{n}\right|}{r_{n}},
$$

i.e., (4.17) holds.

We can now easily show that all pretangent spaces $\Omega_{0, \tilde{r}}^{S}$ are isometric to $S$ and tangent. Indeed, as has been shown above the mapping $F: \tilde{S}_{0, \tilde{r}} \rightarrow S$ is distancepreserving and onto. Hence, by Lemma 4.3 there is an isometry $f: \Omega_{0, \tilde{r}}^{S} \rightarrow S$. Furthermore, statement (iv) of Proposition 1.5 asserts that all $\Omega_{0, \tilde{r}}^{S}$ are tangent if and only if the mappings $\operatorname{in}_{r}^{\prime}: \tilde{S}_{0, \tilde{r}} \rightarrow \tilde{S}_{0, \tilde{r}^{\prime}}$ are surjective for every $\tilde{r}^{\prime}$, see diagram (1.4). 
Let $A$ be an infinite subset of $\mathbf{N}, \tilde{r}^{\prime}=\left\{r_{n}\right\}_{n \in A}$ a subsequence of $\tilde{r}=\left\{r_{n}\right\}_{n \in \mathbf{N}}$ and let $\tilde{y}=\left\{y_{n}\right\}_{n \in A} \in \tilde{S}_{0, \tilde{r}}$. Let us define $x_{n}, n \in \mathbf{N}$, as

$$
x_{n}= \begin{cases}y_{n} & \text { if } n \in A, \\ \frac{y}{r_{n}^{0}} & \text { if } n \in \mathbf{N} \backslash A,\end{cases}
$$

where $r_{n}^{0}$ is defined by (4.18) and $y$ is a point of $S$ such that $|y|=\tilde{d}_{\tilde{r}^{\prime}}(\tilde{y}, \tilde{0})$. It follows from Lemma 4.2 that $\tilde{x}=\left\{x_{n}\right\}_{n \in \mathbf{N}}$ belongs to $\tilde{S}$. Moreover, (4.18) and (4.24) imply

$$
\lim _{n \rightarrow \infty} \frac{d\left(x_{n}, 0\right)}{r_{n}}=|y|<\infty .
$$

Consequently, by Lemma 2.7, we obtain $\tilde{x} \in \tilde{S}_{0, \tilde{r}}$. It is also clear that $\tilde{x}^{\prime}:=\left\{x_{n}\right\}_{n \in A}=$ $\tilde{y}$. Hence each $\operatorname{in}_{r}^{\prime}$ is a surjective mapping.

Remark 4.4. The form of Lemma 4.2 seems to be new. Of course, it is closely related to the contemporary definition of logarithmic spirals, see, for example, [5, 9.6.9.1]. In the proof of Theorem 4.1 we have used formula (4.6) for the verification of condition (i) of Theorem 2.4 but to this end we really need only in the following remarkable fact discovered by Descartes. The length measured along the logarithmic spiral from the pole $O$ to the point $P$ of the spiral is proportional to the radius vector $O P$.

The logarithmic spirals and the set $\mathbf{R}_{+}^{*}:=\mathbf{R}^{+} \backslash\{0\}$ of all strictly positive real numbers have some common properties: They are the unbounded subgroups of the multiplicative group $\mathbf{C}^{*}$ and they have unique pretangent spaces at 0 . The following theorem shows that logarithmic spirals and $\mathbf{R}_{+}^{*}$ are exhausted all maximal subgroups of $\mathbf{C}^{*}$ having these properties.

Theorem 4.5. Let $\Gamma^{*}$ be an unbounded subgroup of the multiplicative group $\mathbf{C}^{*}$ and let $\Gamma:=\Gamma^{*} \cup\{0\}$. The following two statements are equivalent.

(i) $\Gamma^{*} \subseteq \mathbf{R}_{+}^{*}$ or there is $\left.b \in\right] 0,1[\cup] 1, \infty\left[\right.$ such that $\Gamma^{*} \subseteq S^{*}(b)$.

(ii) $(\Gamma, 0) \in \mathfrak{U}$.

Proof. First of all we claim that the theorem is true for all unbounded subgroups $\Gamma^{*}$ if and only if it is true for all closed (in $\mathbf{C}^{*}$ ) unbounded subgroups $\Gamma^{*}$. Let $\bar{\Gamma}^{*}$ be the closure of $\Gamma^{*}$ in $\mathbf{C}^{*}$. For convenience we denote by $\overline{(\mathrm{i})}$ and $\overline{(\mathrm{ii})}$ the results of the substitution of $\bar{\Gamma}^{*}$ for $\Gamma^{*}$ in (i) and, respectively, of $\bar{\Gamma}^{*} \cup\{0\}$ for $\Gamma$ in (ii). Since $\mathbf{R}_{+}^{*}$ and $S^{*}(b)$ are closed subsets of $\mathbf{C}^{*}$ we have the equivalences

$$
\left(\bar{\Gamma}^{*} \subseteq \mathbf{R}_{+}^{*}\right) \Leftrightarrow\left(\Gamma^{*} \subseteq \mathbf{R}_{+}^{*}\right), \quad\left(\bar{\Gamma}^{*} \subseteq S^{*}(b)\right) \Leftrightarrow\left(\Gamma^{*} \subseteq S^{*}(b)\right) .
$$

Thus (i) $\Leftrightarrow \overline{(\mathrm{i})}$. Note now that $\bar{\Gamma}^{*} \cup\{0\}$ is the closure of $\Gamma$ in C. Hence using Lemma 3.3 we see that $\Gamma$ and $\bar{\Gamma} \cup\{0\}$ are strongly tangent equivalent at 0 . By

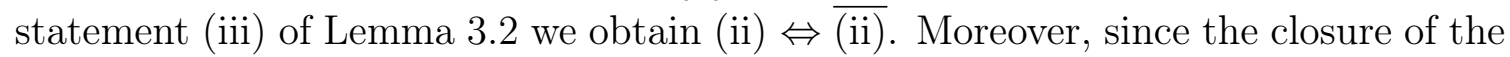
subgroup is a subgroup [19, p. 111], we see that $\bar{\Gamma}^{*}$ is a closed subgroup of $\mathbf{C}^{*}$.

Thus we may assume, without loss of generality, that $\Gamma^{*}$ is a closed subgroup of $\mathrm{C}^{*}$. Let us prove now the implication

(i) $\Rightarrow$ (ii) This implication follows from Proposition 2.9, Theorem 4.1 and Proposition 2.2. Indeed, Proposition 2.2 means, in particular, that $\mathbf{R}^{+}$has unique pretangent spaces at 0 , by Theorem 4.1 the same property is true for $S^{*}(b)$. Finally, the property to have unique pretangent spaces is hereditary by Proposition 2.9. 
(ii) $\Rightarrow$ (i) Suppose that $\Gamma^{*}$ is a closed subgroup of $\mathbf{C}^{*}$ and statement (ii) of the theorem holds. Since $\Gamma^{*}$ is unbounded, there exists a sequence $\left\{p_{i}\right\}_{i \in \mathbf{N}} \in \tilde{\Gamma}^{*}$ with

$$
\lim _{i \rightarrow \infty}\left|p_{i}\right|=\infty
$$

Let $z_{1}, z_{2} \in \Gamma^{*}$ and $\left|z_{1}\right|=\left|z_{2}\right|$. Write $r_{i}=\frac{\left|x_{1}\right|}{\left|p_{i}\right|}$ for $i \in \mathbf{N}$. Note that $\frac{z_{1}}{p_{i}}$ and $\frac{z_{2}}{p_{i}}$ belongs to $\Gamma^{*}$ because $\Gamma^{*}$ is a subgroup of $\mathbf{C}^{*}$. It is clear that

$$
\frac{z_{1}}{p_{i}}, \frac{z_{2}}{p_{i}} \in A_{0}\left(r_{i}, k\right)=\left\{z \in \Gamma: \frac{r_{i}}{k} \leq|z| \leq k r_{i}\right\}
$$

for all $k \in[1, \infty[$ and all $i \in \mathbf{N}$. Statement (ii) of the present theorem implies relation (2.4). Consequently,

$$
0=\lim _{k \rightarrow 1} \lim _{\sup _{i \rightarrow \infty}} \frac{\operatorname{diam}\left(A_{0}\left(r_{i}, k\right)\right)}{r_{i}} \geq \frac{\left|\frac{z_{1}}{p_{i}}-\frac{z_{2}}{p_{i}}\right|}{\left|\frac{z_{1}}{p_{i}}\right|}=\frac{\left|z_{1}-z_{2}\right|}{\left|z_{1}\right|},
$$

i.e., the implication

$$
\left(\left|z_{1}\right|=\left|z_{2}\right|\right) \Rightarrow\left(z_{1}=z_{2}\right)
$$

is true for all $z_{1}, z_{2} \in \Gamma^{*}$.

Let us consider the continuous homomorphism $\Phi: \Gamma^{*} \rightarrow \mathbf{R}_{+}^{*}$ such that

$$
\Phi(z)=|z|, \quad z \in \Gamma^{*} .
$$

It is easy to prove that $\Phi$ is closed. Consequently, $\Phi\left(\Gamma^{*}\right)$ is a closed subgroup of $\mathbf{R}_{+}^{*}$. Using the well-known classification of the closed subgroups of the additive group $\mathbf{R}$, see, for example, $[6$, Chapter $\mathrm{V}, \S 1,1]$, we obtain at the most three possible cases:

(i) $\Phi\left(\Gamma^{*}\right)=\{1\}$,

(ii) $\Phi\left(\Gamma^{*}\right)=\mathbf{R}_{+}^{*}$,

(iii) there is $g \in] 1, \infty\left[\right.$ such that $\Phi\left(\Gamma^{*}\right)=\left\{g^{n}: n \in \mathbf{Z}\right\}$ where $\mathbf{Z}$ is the set of integers.

Since $\Gamma^{*}$ is unbounded, the case (i) is impossible. Implication (4.25) shows that the homomorphism $\Phi: \Gamma^{*} \rightarrow \mathbf{R}_{+}^{*}$ is one-to-one. Consequently, in the case (iii), the group $\Gamma^{*}$ is cyclic with the generator $z=\Phi^{-1}(g)$. Writing $z$ in the trigonometric form $z=|z| e^{i \varphi}=g e^{i \varphi}, \varphi \in\left[0,2 \pi\left[\right.\right.$, we see that either $\Gamma^{*} \subseteq \mathbf{R}_{+}^{*}$ for $\varphi=0$ or, for $\varphi \in] 0,2 \pi\left[, \Gamma^{*}\right.$ lies on the logarithmic spiral

$$
S^{*}=\left\{t \exp \left(i \log _{b} t\right): t \in \mathbf{R}_{+}^{*}\right\}
$$

with $b=\exp \left(\frac{\ln g}{\varphi}\right)$.

Suppose now that we have $\Phi\left(\Gamma^{*}\right)=\mathbf{R}_{+}^{*}$. In this case $\Phi$ is an isomorphism of the groups $\Gamma^{*}$ and $\mathbf{R}_{+}^{*}$ and, simultaneously, $\Phi$ is a homeomorphism as a continuous closed bijection of the topological spaces $\Gamma^{*}$ and $\mathbf{R}_{+}^{*}$. Write $\Phi^{-1}$ for the inverse mapping. Let $\mathbf{T}=\{x \in \mathbf{C}:|z|=1\}$ and $\Psi: \mathbf{C}^{*} \rightarrow \mathbf{T}$ be the standard homomorphism, $\Psi(z)=\frac{z}{|z|}$ and in: $\Gamma^{*} \rightarrow \mathbf{C}^{*}$ be the inclusion in $(z)=z$. Then the mapping

$$
\mathbf{R} \stackrel{\exp }{\longrightarrow} \mathbf{R}_{+}^{*} \stackrel{\Phi^{-1}}{\longrightarrow} \Gamma^{*} \stackrel{\text { in }}{\longrightarrow} \mathbf{C}^{*} \stackrel{\Psi}{\rightarrow} \mathbf{T}
$$

is a character (a continuous homomorphism) of the additive group $\mathbf{R}$. Denote this character by $\varkappa$. There is $\nu \in \mathbf{R}$ such that

$$
\varkappa(t)=\exp (i \nu t)
$$


for all $t \in \mathbf{R}$, see [19, p. 256]. Since $\Phi^{-1}$ is bijective we obtain from (4.26) and (4.27) that

that is

$$
\varkappa(t)=\frac{\Phi^{-1}(\exp t)}{\exp t}=\exp (i \nu t), \quad t \in \mathbf{R}
$$

$$
\begin{gathered}
\frac{\Phi^{-1}(|z|)}{|z|}=\exp (i \nu \ln |z|), \\
z=|z| \exp (i \nu \ln |z|)
\end{gathered}
$$

for all $z \in \Gamma^{*}$. The last equation implies $\Gamma^{*} \subseteq \mathbf{R}_{+}^{*}$ if $\nu=0$. For $\nu \neq 0$ we can rewrite (4.28) as

$$
z=|z| \exp \left(i \log _{b}|z|\right)
$$

where $b=\exp \left(\frac{1}{\nu}\right)$. Hence $\Gamma^{*}$ is the logarithmic spiral.

\section{Uniqueness of pretangent spaces to subsets of $R$}

In this part of the paper we specify the general uniqueness conditions, presented by Theorem 2.4 for arbitrary metric spaces $X$, to the case where $X \subseteq \mathbf{R}$.

Lemma 5.1. Let $X \subseteq \mathbf{R}$ and let $a$ be a limit point of $X$. Condition (i) of Theorem 2.4 does not hold for the pointed metric space $(X, a)$ if and only if there exist some sequences $\tilde{x}=\left\{x_{n}\right\}_{n \in \mathbf{N}}, \tilde{y}=\left\{y_{n}\right\}_{n \in \mathbf{N}}$ belonging to $\tilde{X}$ such that

$$
\left.\lim _{n \rightarrow \infty} x_{n}=\lim _{n \rightarrow \infty} y_{n}=a \quad \text { and } \quad x_{n} \in\right]-\infty, a\left[, y_{n} \in\right] a,+\infty[
$$

for all $n \in \mathbf{N}$ and

$$
\lim _{n \rightarrow \infty} \frac{a-x_{n}}{y_{n}-a}=1
$$

Proof. Let $\tilde{x}=\left\{x_{n}\right\}_{n \in \mathbf{N}}$ and $\tilde{y}=\left\{y_{n}\right\}_{n \in \mathbf{N}}$ satisfy relations (5.1)-(5.2). For every $n \in \mathbf{N}$ write

$$
r=r(n):=\sqrt{\left(a-x_{n}\right)\left(y_{n}-a\right)} \quad \text { and } \quad k=k(n):=\left(\frac{a-x_{n}}{y_{n}-a}\right)^{\frac{1}{2}} \vee\left(\frac{y_{n}-a}{a-x_{n}}\right)^{\frac{1}{2}} .
$$

We can simply show that

$$
\lim _{n \rightarrow \infty} r(n)=0 \quad \text { and } \quad \lim _{n \rightarrow \infty} k(n)=1
$$

and $r(n)>0, k(n) \geq 1$ and

$$
x_{n}, y_{n} \in A_{a}(r(n), k(n))=\left\{x \in X: \frac{r(n)}{k(n)} \leq|x-a| \leq r(r) k(n)\right\}
$$

for all $n \in \mathbf{N}$. Consequently,

$$
\begin{aligned}
\frac{\operatorname{diam}\left(A_{a}(r(n), k(n))\right)}{r(n)} & \geq \frac{\left|y_{n}-x_{n}\right|}{r(n)}=\frac{\left(y_{n}-a\right)+\left(a-x_{n}\right)}{r(n)} \\
& =\sqrt{\frac{y_{n}-a}{a-x_{n}}}+\sqrt{\frac{a-x_{n}}{y_{n}-a}} \geq 2 .
\end{aligned}
$$

Hence the limit relation

$$
\lim _{k \rightarrow 1} \limsup _{r \rightarrow 0} \frac{A_{a}(r, k)}{r} \geq 2
$$

holds. Thus condition (i) of Theorem 2.4 does not hold. 
Suppose now that

$$
\lim _{k \rightarrow 1} \limsup _{r \rightarrow 0} \frac{\operatorname{diam}\left(A_{a}(r, k)\right)}{r}>0 .
$$

We must find the sequences $\tilde{x}=\left\{x_{n}\right\}_{n \in \mathbf{N}} \in \tilde{X}$ and $\tilde{y}=\left\{y_{n}\right\}_{n \in \mathbf{N}} \in \tilde{X}$ which satisfy relations (5.1)-(5.2). Inequality (5.3) implies that there are constant $c>0$ and the sequences $\{r(n)\}_{n \in \mathbf{N}},\{k(n)\}_{n \in \mathbf{N}}$ such that $r(n)>0, k(n) \geq 1$ and $r(n) \downarrow 0, k(n) \downarrow 1$ and

$$
\frac{\operatorname{diam} A_{a}(r(n), k(n))}{r(n)}>c
$$

for all $n \in \mathbf{N}$. Let us consider the closed intervals

$$
I_{n}^{+}:=\left[a+\frac{r(n)}{k(n)}, a+r(n) k(n)\right], \quad I_{n}^{-}:=\left[a-r(n) k(n), a-\frac{r(n)}{k(n)}\right] .
$$

It is clear that

$$
A_{a}(r(n), k(n)) \subseteq I_{n}^{+} \cup I_{n}^{-} .
$$

Inequality (5.4) implies that for every $n \in \mathbf{N}$ there are $x_{n}, y_{n} \in A_{a}(r(n), k(n))$ such that $x_{n}<y_{n}$ and

$$
\frac{\left|x_{n}-y_{n}\right|}{r(n)}>c
$$

If $x_{n}, y_{n} \in I_{n}^{+}$or $x_{n}, y_{n} \in I_{n}^{-}$, then

$$
\frac{\left|x_{n}-y_{n}\right|}{r(n)} \leq k(n)-\frac{1}{k(n)} .
$$

Since $\lim _{n \rightarrow \infty} k(n)=1$, inequality (5.6) contradicts (5.5) for sufficiently large $n$. Using the inequality $x_{n}<y_{n}$ we obtain

$$
x_{n} \in I_{n}^{-} \quad \text { and } \quad y_{n} \in I_{n}^{+},
$$

if $n$ is taken large enough. Relations (5.7) and $\lim _{n \rightarrow \infty} k(n)=1$ imply (5.2). The rest desirable properties of $\tilde{x}=\left\{x_{n}\right\}_{n \in \mathbf{N}}$ and $\tilde{y}=\left\{y_{n}\right\}_{n \in \mathbf{N}}$ are evident from the construction.

Consider now the "real-valued" variants of condition (ii) from Theorem 2.4. Recall that

$$
S_{a}(r)=\{x \in X: d(x, a)=r\} \quad \text { and } \quad R_{a, X}=\left\{r \in \mathbf{R}^{+}: S_{a}(r) \neq \emptyset\right\}
$$

for every pointed metric space $(X, a, d)$. In the case $X \subseteq \mathbf{R}$ there exists the natural decomposition of the set $R_{a, X}$ into the sets

$$
\begin{aligned}
& { }_{1} R_{a, x}:=\left\{r \in R_{a, X}: \operatorname{card} S_{a}(r)=1\right\}, \\
& { }_{2} R_{a, x}:=\left\{r \in R_{a, X}: \operatorname{card} S_{a}(r)=2\right\} .
\end{aligned}
$$

Now we have

$$
R_{a, X}={ }_{1} R_{a, X} \cup{ }_{2} R_{a, X} \quad \text { and } \quad{ }_{1} R_{a, X} \cap{ }_{2} R_{a, X}=\emptyset .
$$

The sets ${ }_{1} R_{a, X}$ and ${ }_{2} R_{a, X}$ are closely related to the symmetric properties of $X \subseteq \mathbf{R}$ in the point $a$. We shall say that $a \in \mathbf{R}$ is a local asymmetry point for the set $X \subseteq \mathbf{R}$ if there is $\varepsilon>0$ so that

$$
(x+a \in X) \Rightarrow(-x+a \notin X)
$$


for all $x$ with $0<|x|<\varepsilon$. The last requirement can be written as

$$
\left.{ }_{2} R_{a, X} \cap\right] 0, \varepsilon\left[=\emptyset \quad \text { or as } \quad{ }_{1} R_{a, X} \cap\right] 0, \varepsilon\left[=R_{a, X} \cap\right] 0, \varepsilon[.
$$

Corollary 5.2. Let $X \subseteq \mathbf{R}$ and let $a$ be a limit point of $X$. If condition (i) of Theorem 2.4 holds, then $a$ is a local asymmetry point for $X$.

Let $X \subseteq \mathbf{R}$ and $a \in X$. For every $r \geq 0$ define

$$
\left(r \in{ }_{+1} R_{a, X}\right) \quad \text { iff } \quad\left(r \in{ }_{1} R_{a, X} \text { and } a+r \in X\right)
$$

and

$$
\left(r \in{ }_{-1} R_{a, X}\right) \quad \text { iff } \quad\left(r \in{ }_{1} R_{a, X} \text { and } a-r \in X\right) .
$$

Then we obtain

$$
{ }_{1} R_{a, X}={ }_{+1} R_{a, X} \cup{ }_{-1} R_{a, X} \text { and } \quad{ }_{+1} R_{a, X} \cap{ }_{-1} R_{a, X}=\{0\},
$$

cf. (5.9). The simple geometric considerations show, see Figure 2, that, for every $q, t \in R_{a, X}$, we have

$$
\Delta\left(S_{a}(q), S_{a}(t)\right)= \begin{cases}|q-t| & \text { if }(q, t) \in\left({ }_{+1} R_{a, X}^{2}\right) \cup\left({ }_{-1} R_{a, X}^{2}\right), \\ |q+t| & \text { otherwise, }\end{cases}
$$

where ${ }_{+1} R_{a, X}^{2}$ and ${ }_{-1} R_{a, X}^{2}$ are the Cartesian squares of ${ }_{+1} R_{a, X}$ and, respectively, of ${ }_{-} R_{a, X}$ and, as usual

$$
\Delta\left(S_{a}(q), S_{a}(t)\right)=\sup \left\{|x-y|: x \in S_{a}(q), y \in S_{a}(t)\right\} .
$$
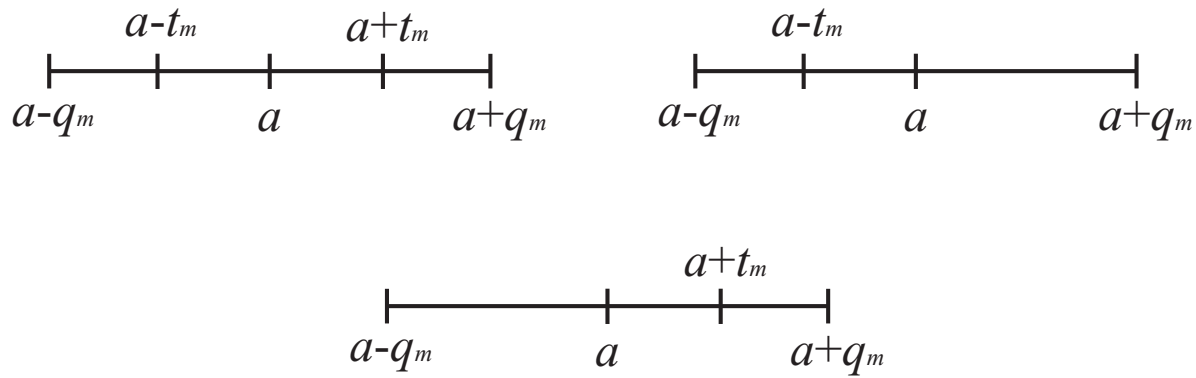

Figure 2. The sphere $S_{a}\left(t_{m}\right)$ lies inside the sphere $S_{a}\left(q_{m}\right)$.

For every $\varepsilon>0$ let us introduce also the sets

$$
\begin{aligned}
{ }_{+} K_{\varepsilon}: & =\left\{\frac{q}{t}: q, t \in{ }_{+1} R_{a, X} \cap[0, \varepsilon]\right\}, \quad{ }_{-} K_{\varepsilon}:=\left\{\frac{q}{t}: q, t \in{ }_{-1} R_{a, X} \cap[0, \varepsilon]\right\}, \\
{ }_{1} K_{\varepsilon}: & =\left\{\frac{q}{t}:(q, t) \in\left(R_{a, X}^{2} \cap[0, \varepsilon]^{2}\right) \backslash\left({ }_{+1} R_{a, X}^{2} \cup{ }_{-1} R_{a, X}^{2}\right)\right\},
\end{aligned}
$$

where $[0, \varepsilon]^{2}$ is the Cartesian product of the closed interval $[0, \varepsilon]$ with itself and

$$
\frac{q}{t}:=\infty \text { if } t=0 .
$$

Proposition 5.3. Let $X$ be a subset of $\mathbf{R}$ and let $a$ be a limit point of $X$. Condition (ii) of Theorem 2.4 holds if and only if

$$
\bigcap_{\varepsilon \in \mathbf{R}_{+}^{*}}\left(C l\left({ }_{+} K_{\varepsilon} \cup{ }_{-} K_{\varepsilon}\right) \cap C l\left({ }_{1} K_{\varepsilon}\right)\right) \subseteq\{0,1, \infty\},
$$

where the closures are taken with respect to $[0, \infty]$. 
Proof. Suppose inclusion (5.13) holds. We must prove condition (ii) of Theorem 2.4. Let $\varepsilon_{0}>0$ and let $\left\{\left(q_{n}, t_{n}\right)\right\}_{n \in \mathbf{N}}$ be a sequence belonging to $\tilde{R}_{\varepsilon_{0}}^{2}$, see Figure 1 , such that $\lim _{n \rightarrow \infty}\left(q_{n}, t_{n}\right)=(0,0)$ and

$$
\lim _{n \rightarrow \infty} \frac{q_{n}}{t_{n}}:=c_{0} \in[0, \infty] .
$$

It is necessary to show that there is a finite limit

$$
\lim _{n \rightarrow \infty} \frac{\Delta\left(S_{a}\left(q_{n}\right), S_{a}\left(t_{n}\right)\right)}{\left|q_{n}-t_{n}\right|}=\varkappa_{0} .
$$

We first note that (5.15) holds with $\varkappa_{0}=1$ if $c_{0}=0$ or $c_{0}=\infty$. Indeed, equality (5.11) implies the double estimation

$$
\frac{\left|q_{n}-t_{n}\right|}{\left|q_{n}-t_{n}\right|} \leq \frac{\Delta\left(S_{a}\left(q_{n}\right), S_{a}\left(t_{n}\right)\right)}{\left|q_{n}-t_{n}\right|} \leq \frac{\left|q_{n}+t_{n}\right|}{\left|q_{n}-t_{n}\right|}
$$

Letting $n \rightarrow \infty$ and using (5.14) with $c_{0} \in\{0, \infty\}$ we obtain (5.15) with $\varkappa_{0}=1$. Moreover, the relations $\left(q_{n}, t_{n}\right) \in R_{\varepsilon_{0}}^{2}$ imply the inequality

$$
\left|\frac{q_{n}}{t_{n}}-1\right| \geq \varepsilon_{0}
$$

so that

$$
c_{0} \neq 1
$$

and $\varkappa_{0}$ is finite, if it exists. Let us consider now the case $0<c_{0}<\infty$. Define, for $\varepsilon>0$,

$$
K_{\varepsilon}:=\left\{\frac{q}{t}: q, t \in R_{a, X} \cap[0, \varepsilon]\right\} .
$$

Then, using the standard representation from the theory of cluster sets, see, for example, $[8,1.1]$, we have

$$
c_{0} \in \bigcap_{\varepsilon \in \mathbf{R}_{+}^{*}} C l\left(K_{\varepsilon}\right) .
$$

Furthermore, it follows from (5.12) that

$$
K_{\varepsilon}={ }_{1} K_{\varepsilon} \cup\left({ }_{+} K_{\varepsilon} \cup{ }_{-} K_{\varepsilon}\right) .
$$

Thus $C l\left(K_{\varepsilon}\right)=C l\left({ }_{1} K_{\varepsilon}\right) \cup C l\left({ }_{+} K_{\varepsilon} \cup{ }_{-} K_{\varepsilon}\right)$. The last equality and the monotonicity: if $\varepsilon_{1} \geq \varepsilon_{2}$, then

$$
C l\left({ }_{1} K_{\varepsilon_{1}}\right) \supseteq C l\left({ }_{1} K_{\varepsilon_{2}}\right) \text { and } C l\left({ }_{+} K_{\varepsilon_{1}} \cup{ }_{-} K_{\varepsilon_{1}}\right) \supseteq C l\left({ }_{+} K_{\varepsilon_{2}} \cup{ }_{-} K_{\varepsilon_{2}}\right)
$$

imply the equality

$$
\bigcap_{\varepsilon \in \mathbf{R}_{+}^{*}} C l\left(K_{\varepsilon}\right)=\left(\bigcap_{\varepsilon \in \mathbf{R}_{+}^{*}} C l\left({ }_{1} K_{\varepsilon}\right)\right) \cup\left(\bigcap_{\varepsilon \in \mathbf{R}_{+}^{*}} C l\left({ }_{+} K_{\varepsilon} \cup{ }_{-} K_{\varepsilon}\right)\right) .
$$

Hence we have

$$
c_{0} \in \bigcap_{\varepsilon \in \mathbf{R}_{+}^{*}} C l\left({ }_{1} K_{\varepsilon}\right)
$$

or

$$
c_{0} \in \bigcap_{\varepsilon \in \mathbf{R}_{+}^{*}} C l\left({ }_{+} K_{\varepsilon} \cup{ }_{-} K_{\varepsilon}\right) .
$$


It follows directly form (5.13) that

$$
\left(\bigcap_{\varepsilon \in \mathbf{R}_{+}^{*}} C l\left({ }_{1} K_{\varepsilon}\right)\right) \cap\left(\bigcap_{\varepsilon \in \mathbf{R}_{+}^{*}} C l\left({ }_{+} K_{\varepsilon} \cup{ }_{-} K_{\varepsilon}\right)\right) \subseteq\{0,1, \infty\} .
$$

Since $c_{0} \notin\{0,1, \infty\}$, representation (5.18) and (5.19) imply that there is $n_{0} \in \mathbf{N}$ such that either

$$
\left(q_{n}, t_{n}\right) \in R_{a, X}^{2} \backslash\left({ }_{+1} R_{a, X}^{2} \cup{ }_{-1} R_{a, X}^{2}\right)
$$

for all $n \geq n_{0}$, or

$$
\left(q_{n}, t_{n}\right) \in{ }_{+1} R_{a, X}^{2} \cup{ }_{-1} R_{a, X}^{2}
$$

for all $n \geq n_{0}$. Now applying (5.11) we obtain

$$
\varkappa_{0}= \begin{cases}\frac{1+c_{0}}{\left|1-c_{0}\right|} & \text { if }(5.20) \text { holds } \\ 1 & \text { if }(5.21) \text { holds }\end{cases}
$$

The "sufficiency" is proved.

To prove the "necessity" suppose that (5.13) does not hold. The left side of (5.13) can be written as

$$
\left(\bigcap_{\varepsilon \in \mathbf{R}_{+}^{*}} C l\left({ }_{+} K_{\varepsilon} \cup{ }_{-} K_{\varepsilon}\right)\right) \cap\left(\bigcap_{\varepsilon \in \mathbf{R}_{+}^{*}} C l\left({ }_{1} K_{\varepsilon}\right)\right) .
$$

Consequently there is $\left.c_{0} \in\right] 0, \infty\left[\right.$ such that $c_{0} \neq 1$ and

$$
c_{0} \in \bigcap_{\varepsilon \in \mathbf{R}_{+}^{*}} C l\left({ }_{+} K_{\varepsilon} \cup{ }_{-} K_{\varepsilon}\right) \quad \text { and } \quad c_{0} \in \bigcap_{\varepsilon \in \mathbf{R}_{+}^{*}} C l\left({ }_{1} K_{\varepsilon}\right) .
$$

Hence there are two sequences $\left\{\left(z_{n}, w_{n}\right)\right\}_{n \in \mathbf{N}}$ and $\left\{\left(q_{n}, t_{n}\right)\right\}_{n \in \mathbf{N}}$ such that

$$
\left(z_{n}, w_{n}\right) \in\left({ }_{+1} R_{a, X}^{2} \cup{ }_{-1} R_{a, X}^{2}\right)
$$

and

$$
\left(q_{n}, t_{n}\right) \in\left(R_{a, X}^{2}\right) \backslash\left({ }_{+1} R_{a, X}^{2} \cup{ }_{-1} R_{a, X}^{2}\right)
$$

for all $n \in \mathbf{N}$, and

$$
\lim _{n \rightarrow \infty}\left(q_{n}, t_{n}\right)=\lim _{n \rightarrow \infty}\left(z_{n}, w_{n}\right)=(0,0)
$$

and

$$
\lim _{n \rightarrow \infty} \frac{q_{n}}{t_{n}}=\lim _{n \rightarrow \infty} \frac{z_{n}}{w_{n}}=c_{0} .
$$

Since $c_{0} \neq 1$, there is $\varepsilon_{0}>0$ such that

$$
\left|\frac{q_{n}}{t_{n}}-1\right| \geq \varepsilon_{0} \quad \text { and } \quad\left|\frac{z_{n}}{w_{n}}-1\right| \geq \varepsilon_{0}
$$

if $n$ is sufficiently large. Equality (5.11) and (5.22), (5.23) imply

$$
\lim _{n \rightarrow \infty} \frac{\Delta\left(S_{a}\left(q_{n}\right), S_{a}\left(t_{n}\right)\right)}{\left|q_{n}-t_{n}\right|}=\frac{1+c_{0}}{\left|1-c_{0}\right|}
$$

and

$$
\lim _{n \rightarrow \infty} \frac{\Delta\left(S_{a}\left(z_{n}\right), S_{a}\left(w t_{n}\right)\right)}{\left|z_{n}-w_{n}\right|}=1 .
$$


Let $n_{0}$ be a natural number such that (5.26) holds for all $n \geq n_{0}$. Define a sequence $\left\{\left(s_{n}, y_{n}\right)\right\}_{n \in \mathbf{N}}$ as the "mixture" of the sequences $\left\{\left(q_{n}, t_{n}\right)\right\}_{n \in \mathbf{N}}$ and $\left\{\left(z_{n}, w_{n}\right)\right\}_{n \in \mathbf{N}}$,

$$
\left(s_{n}, y_{n}\right)= \begin{cases}\left(q_{n_{0}}, t_{n_{0}}\right) & \text { if } n \leq n_{0}, \\ \left(q_{n}, t_{n}\right) & \text { if } n \text { is add and } n>n_{0}, \\ \left(z_{n}, w_{n}\right) & \text { if } n \text { is even and } n>n_{0} .\end{cases}
$$

Then $\left(s_{n}, y_{n}\right) \in R_{\varepsilon_{0}}^{2}$ for all $n \in \mathbf{N}$ and, by (5.24), (5.25), we have

$$
\lim _{n \rightarrow \infty}\left(s_{n}, y_{n}\right)=(0,0), \quad \lim _{n \rightarrow \infty} \frac{s_{n}}{y_{n}}=c_{0} .
$$

If there is the limit

$$
\lim _{n \rightarrow \infty} \frac{\Delta\left(S_{a}\left(s_{n}\right), S_{a}\left(y_{n}\right)\right)}{\left|s_{n}-y_{n}\right|},
$$

then the definition of the sequence $\left\{\left(s_{n}, y_{n}\right)\right\}_{n \in \mathbf{N}}$ and equalities (5.27), (5.28) imply

$$
1=\frac{1+c_{0}}{\left|1-c_{0}\right|} \text {. }
$$

Hence $c_{0}=0$, contrary to the condition $c_{0} \notin\{0,1, \infty\}$.

Thus it is proved that condition (ii) of Theorem 2.4 does not hold if (5.13) is false.

The following theorem is the main result of the present section of the paper. Define, for $\varepsilon>0$,

$$
{ }_{+-} K_{\varepsilon}:=\left\{\frac{q}{t}: q \in{ }_{+1} R_{a, X} \cap[0, \varepsilon], t \in{ }_{-1} R_{a, X} \cap[0, \varepsilon]\right\},
$$

where $\frac{q}{t}:=\infty$ if $t=0$.

Theorem 5.4. Let $X$ be subset of $\mathbf{R}$ and let a be a limit point of $X$. Then for every normalizing sequence $\tilde{r}$ there is a unique pretangent space $\Omega_{a, \tilde{r}}^{X}$ if and only if $X$ is locally asymmetric at the point $a$ and

$$
\bigcap_{\varepsilon \in \mathbf{R}_{+}^{*}}\left(C l\left({ }_{+} K_{\varepsilon} \cup{ }_{-} K_{\varepsilon}\right) \cap C l\left(_{+-} K_{\varepsilon}\right)\right) \subseteq\{0, \infty\} .
$$

Proof. Suppose $(X, a) \in \mathfrak{U}$. Then conditions (i)-(ii) of Theorem 2.4 holds. Using Corollary 5.2 we see that $a$ is the local asymmetry point for the set $X$. Moreover, since ${ }_{1} K_{\varepsilon} \supseteq{ }_{+-} K_{\varepsilon}$, we obtain from inclusion (5.13) the inclusion

$$
\bigcap_{\varepsilon \in \mathbf{R}_{+}^{*}}\left(C l\left({ }_{+} K_{\varepsilon} \cup{ }_{-} K_{\varepsilon}\right) \cap C l\left(_{+-} K_{\varepsilon}\right)\right) \subseteq\{0,1, \infty\} .
$$

It still remains to prove that

$$
1 \notin \bigcap_{\varepsilon \in \mathbf{R}_{+}^{*}}\left(C l\left(_{+} K_{\varepsilon} \cup{ }_{-} K_{\varepsilon}\right) \cap C l\left(_{+-} K_{\varepsilon}\right)\right) .
$$

Since $X$ is locally asymmetric at $a$ and $a$ is a limit point of $X$, we have

$$
1 \in \bigcap_{\varepsilon \in \mathbf{R}_{+}^{*}} C l\left({ }_{+} K_{\varepsilon} \cup{ }_{-} K_{\varepsilon}\right) .
$$


Taking into account this and the equality

$$
\bigcap_{\varepsilon \in \mathbf{R}_{+}^{*}}\left(C l\left({ }_{+} K_{\varepsilon} \cup_{-} K_{\varepsilon}\right) \cap C l\left({ }_{+-} K_{\varepsilon}\right)\right)=\left(\bigcap_{\varepsilon \in \mathbf{R}_{+}^{*}} C l\left({ }_{+} K_{\varepsilon} \cup{ }_{-} K_{\varepsilon}\right)\right) \cap\left(\bigcap_{\varepsilon \in \mathbf{R}_{+}^{*}} C l\left({ }_{+-} K_{\varepsilon}\right)\right)
$$

we see that (5.31) is equivalent to

$$
1 \notin \bigcap_{\varepsilon \in \mathbf{R}_{+}^{*}} C l\left({ }_{+-} K_{\varepsilon}\right) .
$$

If (5.34) does not hold, then there exist some sequences $\tilde{x}=\left\{x_{n}\right\}_{n \in \mathbf{N}}, \tilde{y}=\left\{y_{n}\right\}_{n \in \mathbf{N}}$ belonging to $\tilde{X}$ such that $\lim _{n \rightarrow \infty} x_{n}=\lim _{n \rightarrow \infty} y_{n}=a$ and $\left.x_{n} \in\right]-\infty, a\left[, y_{n} \in\right] a,+\infty[$ for all $n \in \mathbf{N}$ and

$$
\lim _{n \rightarrow \infty} \frac{a-x_{n}}{y_{n}-a}=1
$$

By Lemma 5.1 it contradicts condition (i) of Theorem 2.4. Thus (5.31) is proved.

Conversely, suppose that $a$ is a local asymmetry point of $X$ and inclusion (5.30) holds. We must prove conditions (i)-(ii) of Theorem 2.4.

Rewriting Lemma 5.1 in the terms of cluster sets we see that condition (i) does not hold if and only if

$$
1 \in \bigcap_{\varepsilon \in \mathbf{R}_{+}^{*}} C l\left({ }_{+-} K_{\varepsilon}\right) .
$$

This relation, (5.32) and (5.33) show that the point 1 belongs to the set in the left part of formula (5.30), contrary to this formula.

To prove condition (ii) of Theorem 2.4 note that

$$
\begin{aligned}
{ }_{1} K_{\varepsilon} & =\left(R_{a, X}^{2} \backslash\left({ }_{+1} R_{a, X}^{2} \cup{ }_{-1} R_{a, X}^{2}\right)\right) \cap[0, \varepsilon]^{2} \\
& =\left({ }_{+1} R_{a, X} \times{ }_{-1} R_{a, X}\right) \cup\left({ }_{-1} R_{a, X} \times{ }_{+1} R_{a, X}\right) \cap[0, \varepsilon]^{2}
\end{aligned}
$$

for sufficiently small $\varepsilon>0$ if $a$ is the local asymmetry point of $X$. Hence, by Proposition 5.3, condition (ii) holds if

$$
\bigcap_{\varepsilon \in \mathbf{R}_{+}^{*}}\left(C l\left({ }_{+} K_{\varepsilon} \cup{ }_{-} K_{\varepsilon}\right) \cap C l\left(_{+-} K_{\varepsilon} \cup{ }_{-+} K_{\varepsilon}\right)\right) \subseteq\{0,1, \infty\} .
$$

Here ${ }_{-+} K_{\varepsilon}$ is the set obtained by the permutation of the symbols + and - in (5.29). Similarly (5.17) we can show that

$$
\begin{gathered}
\bigcap_{\varepsilon \in \mathbf{R}_{+}^{*}}\left(C l\left({ }_{+} K_{\varepsilon} \cup{ }_{-} K_{\varepsilon}\right) \cap C l\left(_{+-} K_{\varepsilon} \cup{ }_{-+} K_{\varepsilon}\right)\right) \\
=\left(\bigcap_{\varepsilon \in \mathbf{R}_{+}^{*}}\left(C l\left(_{+} K_{\varepsilon} \cup{ }_{-} K_{\varepsilon}\right) \cap C l\left({ }_{+} K_{\varepsilon}\right)\right)\right) \\
\cup\left(\bigcap_{\varepsilon \in \mathbf{R}_{+}^{*}}\left(C l\left(_{+} K_{\varepsilon} \cup{ }_{-} K_{\varepsilon}\right) \cap C l\left({ }_{-+} K_{\varepsilon}\right)\right)\right) .
\end{gathered}
$$

It follows from the definition of the sets ${ }_{+} K_{\varepsilon},{ }_{-} K_{\varepsilon},{ }_{+-} K_{\varepsilon}$ and ${ }_{-+} K_{\varepsilon}$ that if a positive number $s$ belongs to

$$
\bigcap_{\varepsilon \in \mathbf{R}_{+}^{*}}\left(C l\left({ }_{+} K_{\varepsilon} \cup{ }_{-} K_{\varepsilon}\right) \cap C l\left({ }_{-+} K_{\varepsilon}\right)\right),
$$


then

$$
\frac{1}{s} \in \bigcap_{\varepsilon \in \mathbf{R}_{+}^{*}}\left(C l\left({ }_{+} K_{\varepsilon} \cup{ }_{-} K_{\varepsilon}\right) \cap C l\left({ }_{+-} K_{\varepsilon}\right)\right) .
$$

Consequently, we can transpose "+" and "-" in inclusion (5.30). Thus we have the inclusion

$$
\bigcap_{\varepsilon \in \mathbf{R}_{+}^{*}}\left(C l\left({ }_{+} K_{\varepsilon} \cup{ }_{-} K_{\varepsilon}\right) \cap C l\left({ }_{+-} K_{\varepsilon} \cup{ }_{-+} K_{\varepsilon}\right)\right) \subseteq\{0, \infty\}
$$

that is stronger than (5.36).

\section{Uniqueness on the Cantor set}

Recall the definition of the Cantor set. Let $x \in[0,1]$ and expand $x$ as

$$
x=\sum_{n=1}^{\infty} \frac{b_{n}(x)}{3^{n}}, \quad b_{n}(x) \in\{0,1,2\} .
$$

The Cantor set $C$ is the set of all points from [0,1] which have expansion (6.1) using only the digits 0 and 2. Thus $x$ belongs to $C$ if and only if $x$ has a triadic representation

$$
x=\sum_{m=1}^{\infty} \frac{2 \alpha_{m}}{3^{m}}
$$

where $\alpha_{m}=\alpha_{m}(x) \in\{0,1\}$.

Denote by $C^{1}$ the set of all endpoints of complementary intervals of $C$ and write $C^{0}:=C \backslash C^{1}$.

Proposition 6.1. Let $X$ be a closed subset of $\mathbf{R}$. If $a \in X$ is an endpoint of a complementary interval of $X$, then $(X, a) \in \mathfrak{U}$.

Proof. Since $a$ is an endpoints of a complementary interval of $X$, there is $\varepsilon>0$ such that either

$$
[a-\varepsilon, a+\varepsilon] \cap X \subseteq]-\infty, a] \quad \text { or } \quad[a-\varepsilon, a+\varepsilon] \cap X \subseteq[a,+\infty[.
$$

Hence $X$ is locally isometric to a subset of $\mathbf{R}_{+}$. Observe that for every two locally isometric pointed metric spaces $(Z, p)$ and $(Y, b)$ the uniqueness of pretangent spaces $\Omega_{p, \tilde{r}}^{Z}$ implies the uniqueness of $\Omega_{b, \tilde{r}}^{Y}$. The last assertion follows directly from the definition of pretangent spaces or, more formally, from Theorem 2.4. Thus the uniqueness of $\Omega_{a, \tilde{r}}^{X}$ follows from Proposition 2.9 and Proposition 2.2.

Corollary 6.2. The relation $(C, a) \in \mathfrak{U}$ holds for each $a \in C^{1}$.

The main purpose of the present section of the paper is to prove the conversion of Corollary 6.2, i.e., $((C, a) \in \mathfrak{U}) \Rightarrow\left(a \in C^{1}\right)$. Moreover, we prove that the Cantor set $C$ is locally asymmetric in each its point. In the end of the section we shall give the explicit form of the pretangent space $\Omega_{a, \tilde{r}}^{C}$ for $a \in C^{1}$ and $\tilde{r}=\left\{3^{-n}\right\}_{n \in \mathbf{N}}$.

Proposition 6.3. All point $a \in C$ are the points of the local asymmetry for $C$.

Proof. It is clear if $a \in C^{1}$. Suppose $a \in C^{0}$ and there are two distinct points $x, y \in C$ such that

$$
a=\frac{x+y}{2} .
$$


Writing $a, x$ and $y$ in form (6.1) we obtain from (6.3) the equality

$$
\sum_{n=1}^{\infty} \frac{b_{n}(a)}{3^{n}}=\sum_{n=1}^{\infty} \frac{\frac{1}{2}\left(b_{n}(y)+b_{n}(x)\right)}{3^{n}},
$$

where $b_{n}(a), b_{n}(x), b_{n}(y) \in\{0,2\}$ for all $n$. Since $x \neq y$, there is $n_{0}$ such that either

$$
b_{n_{0}}(y)=2 \quad \text { and } \quad b_{n_{0}}(x)=0
$$

or

$$
b_{n_{0}}(y)=0 \quad \text { and } \quad b_{n_{0}}(x)=2 .
$$

Thus the number $a \in C$ has two different ternary representations. The point $a$ of the Cantor set $C$ has two different ternary representations if and only if $a \in C^{1} \backslash\{0\}$. (It follows directly from Problem 22 in [20, p. 40]). Consequently, (6.4) contradicts the relation $a \in C^{0}$.

Remark 6.4. A simple result related to the asymmetry of the Cantor set $C$ can be found in [21, p. 50].

Define a subset $C^{2}$ of the Cantor set $C$ by the rule: $x \in C^{2}$ if and only if $x \in C$ and there is $m_{0}=m_{0}(x) \in \mathbf{N}$ such that, for all $m>m_{0}$,

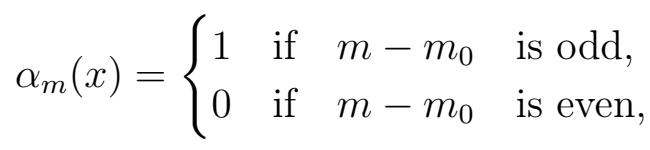

where $\alpha_{m}(x)$ are the digits in ternary representation (6.2). For example, the numbers

$$
\frac{3}{4}=\frac{2}{3}+\frac{0}{3^{2}}+\frac{2}{3^{3}}+\frac{0}{3^{4}}+\ldots
$$

and

$$
\frac{1}{4}=\frac{0}{3}+\frac{2}{3^{2}}+\frac{0}{3^{3}}+\frac{2}{3^{4}}+\ldots
$$

belong to $C^{2}$.

Lemma 6.5. Let $a \in C^{0} \backslash C^{2}$. If ${ }_{+} K_{\varepsilon},{ }_{-} K_{\varepsilon}$ and ${ }_{1} K_{\varepsilon}$ are the sets defined by (5.12), then

$$
3 \in \bigcap_{\varepsilon \in \mathbf{R}_{+}^{*}}\left(C l\left({ }_{+} K_{\varepsilon} \cup{ }_{-} K_{\varepsilon}\right) \cap C l\left({ }_{1} K_{\varepsilon}\right)\right) .
$$

Remark 6.6. Let $x$ and $y$ be distinct points of the Cantor set,

$$
x=\sum_{m=1}^{\infty} \frac{2 \alpha_{m}(x)}{3^{m}}, \quad y=\sum_{m=1}^{\infty} \frac{2 \alpha_{m}(y)}{3^{m}}, \quad \alpha_{m}(x), \alpha_{m}(y) \in\{0,1\} .
$$

Write $M$ for the smallest natural number $m$ such that $\alpha_{m}(x) \neq \alpha_{m}(y)$. Then the inequality $x>y$ holds if and only if $\alpha_{M}(x)=1$ and $\alpha_{M}(y)=0$. It enables us to reformulate the definitions of the sets ${ }_{+} K_{\varepsilon},{ }_{-} K_{\varepsilon}$ etc. for the specific case when $X$ is the Cantor set. For example, a point $s$ belongs to ${ }_{+} K_{\varepsilon}$ if and only if there are $M_{1}, M_{2} \in \mathbf{N}$ and sequences $\left\{\beta_{m}\right\}_{m \in \mathbf{N}},\left\{\gamma_{m}\right\}_{m \in \mathbf{N}}$ with $\beta_{m}, \gamma_{m} \in\{0,1\}$, such that

$$
s=\frac{3^{-M_{1}}+\sum_{m=M_{1}+1}^{\infty} \frac{\beta_{m}-\alpha_{m}(a)}{3^{m}}}{3^{-M_{2}}+\sum_{m=M_{2}+1}^{\infty} \frac{\gamma_{m}-\alpha_{m}(a)}{3^{m}}}
$$


and

$$
\left(3^{-M_{1}}+\sum_{m=M_{1}+1}^{\infty} \frac{\beta_{m}-\alpha_{m}(a)}{3^{m}}\right) \vee\left(3^{-M_{2}}+\sum_{m=M_{2}+1}^{\infty} \frac{\gamma_{m}-\alpha_{m}(a)}{3^{m}}\right) \leq \frac{\varepsilon}{2} .
$$

Proof of Lemma 6.5. Since $a \in C^{0}$, there exists a strictly increasing sequence $\left\{m_{k}\right\}_{k \in \mathbf{N}}$ of natural numbers such that either

$$
\alpha_{m_{k}}(a)=0 \text { and } \alpha_{m_{k}+1}(a)=1 \quad \text { for all } k \in \mathbf{N}
$$

or

$$
\alpha_{m_{k}}(a)=1 \text { and } \alpha_{m_{k}+1}(a)=0 \text { for all } k \in \mathbf{N} .
$$

Suppose (6.8) holds. (The case when (6.9) holds is similar.) Equalities (6.8) imply that

$$
C \ni a+2 \cdot 3^{-m_{k}}>a \text { and } C \ni a-2 \cdot 3^{-\left(m_{k}+1\right)}<a .
$$

Thus

$$
2 \cdot 3^{-m_{k}} \in{ }_{+} R_{a, C} \text { and } 2 \cdot 3^{-\left(m_{k}+1\right)} \in{ }_{-} R_{a, C} .
$$

Hence

$$
\frac{2 \cdot 3^{-m_{k}}}{2 \cdot 3^{-\left(m_{k}+1\right)}}=3 \in{ }_{1} K_{\varepsilon}
$$

for all $\varepsilon>0$. Thus we obtain

$$
3 \in \bigcap_{\varepsilon \in \mathbf{R}_{+}^{*}} C l\left({ }_{1} K_{\varepsilon}\right) .
$$

On the other hand, since $a \in C^{0} \backslash C^{2}$, there is a strictly increasing sequence $\left\{m_{k}\right\}_{k \in \mathbf{N}}$ such that either

$$
\alpha_{m_{k}}(a)=\alpha_{m_{k}+1}(a)=0 \quad \text { for all } k \in \mathbf{N}
$$

or

$$
\alpha_{m_{k}}(a)=\alpha_{m_{k}+1}(a)=1 \quad \text { for all } k \in \mathbf{N} .
$$

Reasoning as in the proof of (6.10) we obtain the membership relation

$$
3 \in \bigcap_{\varepsilon \in \mathbf{R}_{+}^{*}} C l\left({ }_{+} K_{\varepsilon} \cup K_{-}\right) .
$$

It still remains to observe that (6.10) and (6.11) imply (6.7).

Lemma 6.7. Let $X=C$ and $a \in C^{2}$. The following statements hold for every $\gamma \in \mathbf{R}_{+}^{*}$.

(i) The membership relation

$$
\gamma \in \bigcap_{\varepsilon \in \mathbf{R}_{+}^{*}} C l\left({ }_{+} K_{\varepsilon} \cup K_{\varepsilon}\right)
$$

holds if and only if there are $\theta_{1}, \theta_{2} \in C$ and $m \in \mathbf{Z}$ such that

$$
\gamma=3^{2 m} \frac{5+4 \theta_{1}}{5+4 \theta_{2}} .
$$


(ii) The membership relation

$$
\gamma \in \bigcap_{\varepsilon \in \mathbf{R}_{+}^{*}} C l\left({ }_{1} K_{\varepsilon}\right)
$$

holds if and only if there are $\theta_{3}, \theta_{4} \in C$ and $m \in \mathbf{Z}$ such that

$$
\gamma=3^{2 m+1} \frac{5+4 \theta_{3}}{5+4 \theta_{4}} \text {. }
$$

Proof. (i) Let (6.12) hold. Then we obtain

$$
\gamma \in \bigcap_{\varepsilon \in \mathbf{R}_{+}^{*}} C l\left({ }_{+} K_{\varepsilon}\right) \quad \text { or } \quad \gamma \in \bigcap_{\varepsilon \in \mathbf{R}_{+}^{*}} C l\left({ }_{-} K_{\varepsilon}\right) .
$$

Suppose the first relation holds. Then, by the definition of the cluster set $\bigcap_{\varepsilon \in \mathbf{R}_{+}^{*}} C l\left({ }_{+} K_{\varepsilon}\right)$, there are $\left\{x_{n}\right\}_{n \in \mathbf{N}} \in \tilde{X}$ and $\left\{y_{n}\right\}_{n \in \mathbf{N}} \in \tilde{X}$ such that $\lim _{n \rightarrow \infty} x_{n}=\lim _{n \rightarrow \infty} y_{n}=a$ and $a<x_{n} \wedge y_{n}$ for all $n \in \mathbf{N}$ and

$$
\gamma=\lim _{n \rightarrow \infty} \frac{x_{n}-a}{y_{n}-a}
$$

As in (7.9) we define

$$
k^{\prime}=k^{\prime}\left(x_{n}, a\right):=\min \left\{m: \alpha_{m}\left(x_{n}\right) \neq \alpha_{m}(a)\right\}
$$

and

$$
k "=k "\left(x_{n}, a\right):=\min \left\{m: \alpha_{m}\left(y_{n}\right) \neq \alpha_{m}(a)\right\} .
$$

Now the inequality $a<x_{n} \wedge y_{n}$ implies $\alpha_{k^{\prime}}\left(x_{n}\right)=\alpha_{k^{\prime \prime}}\left(y_{n}\right)=1$ and $\alpha_{k^{\prime}}(a)=\alpha_{k^{\prime \prime}}(a)=$ 0 . Furthermore if $n$ is sufficiently large, then

$$
\sum_{m=k^{\prime}+1}^{\infty} \frac{2 \alpha_{m}(a)}{3^{m}}=\left(\frac{2}{3^{k^{\prime}+1}}+\frac{2}{3^{k^{\prime}+3}}+\frac{2}{3^{k^{\prime}+5}}+\ldots\right)=\frac{1}{3^{k^{\prime}}} \frac{3}{4}
$$

and similarly

$$
\sum_{m=k^{\prime \prime}+1}^{\infty} \frac{2 \alpha_{m}(a)}{3^{m}}=\left(\frac{2}{3^{k \prime+1}}+\frac{2}{3^{k \prime}+3}+\frac{2}{3^{k \prime+5}}+\ldots\right)=\frac{1}{3^{k \prime}} \frac{3}{4},
$$

because $\lim _{n \rightarrow \infty} x_{n}=\lim _{n \rightarrow \infty} y_{n}=a$. Consequently, we have

$$
\frac{x_{n}-a}{y_{n}-a}=\frac{\frac{2}{3^{k^{\prime}}}+\frac{1}{3^{k^{\prime}}} \theta\left(x_{n}\right)-\frac{1}{3^{k^{\prime}}} \frac{3}{4}}{\frac{2}{3^{k^{\prime \prime}}}+\frac{1}{3^{k^{\prime \prime}}} \theta\left(y_{n}\right)-\frac{1}{3^{k^{\prime \prime}}} \frac{3}{4}}=\frac{3^{k^{\prime \prime}}}{3^{k^{\prime}}} \frac{\frac{5}{4}+\theta\left(x_{n}\right)}{\frac{5}{4}+\theta\left(y_{n}\right)},
$$

where

$$
\frac{1}{3^{k^{\prime}}} \theta\left(x_{n}\right)=\sum_{m=k^{\prime}+1}^{\infty} \frac{\alpha_{m}\left(x_{n}\right)}{3^{m}} \text { and } \frac{1}{3^{k^{\prime \prime}}} \theta\left(y_{n}\right)=\sum_{m=k^{\prime \prime}+1}^{\infty} \frac{\alpha_{m}\left(y_{n}\right)}{3^{m}} .
$$

Since $C$ is a compact set and $\theta\left(x_{n}\right), \theta\left(y_{n}\right) \in C$, there is a subsequence $\left\{n_{l}\right\}_{l \in \mathbf{N}}$ such that

$$
\lim _{l \rightarrow \infty} \theta\left(x_{n_{l}}\right):=\theta_{1} \quad \text { and } \quad \lim _{l \rightarrow \infty} \theta\left(y_{n_{l}}\right):=\theta_{2}
$$

with $\theta_{1}, \theta_{2} \in C$. These equalities, (6.18) and (6.17) imply the convergence

$$
\gamma \frac{5 / 4+\theta_{2}}{5 / 4+\theta_{1}}=\lim _{l \rightarrow \infty} 3^{k^{\prime \prime}\left(y_{n_{l}}, a\right)-k^{\prime}\left(x_{n_{l}}, a\right)}
$$


Since $\gamma \in \mathbf{R}_{+}^{*}$ and $k^{\prime \prime}\left(y_{n_{l}}, a\right)-k^{\prime}\left(x_{n_{l}}, a\right) \in \mathbf{Z}$, we obtain the equality

$$
\gamma=3^{p} \frac{5+4 \theta_{1}}{5+4 \theta_{2}}
$$

where $p=\lim _{l \rightarrow \infty}\left(k^{\prime \prime}\left(y_{n_{l}}, a\right)-k^{\prime}\left(x_{n_{l}}, a\right)\right) \in \mathbf{Z}$. As was noted above

$$
\alpha_{k^{\prime}}(a)=\alpha_{k^{\prime \prime}}(a)=0 .
$$

Hence from the definition of the set $C^{2}$ and the limit relations

$$
a=\lim _{n \rightarrow \infty} x_{n}=\lim _{n \rightarrow \infty} y_{n}
$$

we obtain that $\left(k^{\prime \prime}\left(y_{n_{l}}, a\right)-k^{\prime}\left(x_{n_{l}}, a\right)\right)$ is even number if $n$ sufficiently large. Thus $p$ in (6.19) is even, i.e., (6.13) is proved for the case when $\gamma \in \bigcap_{\varepsilon \in \mathbf{R}_{+}^{*}} C l\left({ }_{+} K_{\varepsilon}\right)$.

If $\gamma \in \bigcap_{\varepsilon \in \mathbf{R}_{+}^{*}} C l\left({ }_{-} K_{\varepsilon}\right)$, then the proof of $(6.13)$ can be reduced to the previous case by the isometry $x \mapsto 1-x$. Indeed, the sets $C$ and $C^{2}$ are invariant under this isometry and $a<x_{n} \wedge y_{n} \Leftrightarrow(1-a)>\left(1-x_{n}\right) \vee\left(1-y_{n}\right)$ and

$$
\frac{a-x_{n}}{a-y_{n}}=\frac{\left(1-x_{n}\right)-(1-a)}{\left(1-y_{n}\right)-(1-a)} \text {. }
$$

Suppose now that $\gamma$ is a positive real number having form (6.13). We must prove (6.12). Write

$$
l_{0}=l_{0}(a):=2|m|+m_{0}(a)+1,
$$

where $2 m$ is the exponent in (6.13) and $m_{0}(a)$ is the constant from the definition of the set $C^{2}$, see (6.5). Note that

$$
\alpha_{l_{0}-2|m|}(a)=\ldots=\alpha_{l_{0}}(a)=\alpha_{l_{0}+2}(a)=\alpha_{l_{0}+4}(a)=\ldots=0
$$

and

$$
\alpha_{l_{0}-2|m|-1}(a)=\ldots=\alpha_{l_{0}+1}(a)=\alpha_{l_{0}+3}(a)=\alpha_{l_{0}+5}(a)=\ldots=1 .
$$

For every $n \in \mathbf{N}$ define the natural numbers $k^{\prime}$ and $k "$ as

$$
k^{\prime}(n):=l_{0}(a)+2 n-1, k^{\prime \prime}(n):=l_{0}(a)+2 m+2 n-1
$$

and write

$$
x_{n}=\sum_{j=1}^{k^{\prime}(n)} \frac{2 \alpha_{j}(a)}{3^{j}}+\frac{2}{3^{k^{\prime}(n)}}+\frac{\theta_{1}}{3^{k^{\prime}(n)}}, \quad y_{n}=\sum_{j=1}^{k^{\prime \prime}(n)} \frac{2 \alpha_{j}(a)}{3^{j}}+\frac{2}{3^{k^{\prime \prime}(n)}}+\frac{\theta_{2}}{3^{k^{\prime \prime}(n)}},
$$

It is easy to see that $x_{n}, y_{n} \in C$ for all $n$. Using (6.20)-(6.23) we obtain the inequalities $x_{n}>a$ and $y_{n}>a$ and the equalities

$$
\frac{a-x_{n}}{a-y_{n}}=3^{k^{\prime \prime}(n)-k^{\prime}(n)} \frac{5+4 \theta_{1}}{5+4 \theta_{2}}=3^{2 m} \frac{5+4 \theta_{1}}{5+4 \theta_{2}}=\gamma
$$

for every $n \in \mathbf{N}$. Consequently, we have

$$
\gamma \in \bigcap_{\varepsilon \in \mathbf{R}_{+}^{*}} C l\left({ }_{+} K_{\varepsilon}\right)
$$

that implies (6.12). 
(ii) The proof of the second statement of the present lemma is similar to the proof of the first one. It should be noted only that for $x_{n}<a<y_{n}$, we have

$$
\begin{aligned}
\frac{a-x_{n}}{y_{n}-a} & =\frac{\frac{2}{3^{k^{\prime}}}+\left(\frac{0}{3^{k^{\prime}+1}}+\frac{2}{3^{k^{\prime}+2}}+\frac{0}{3^{k^{\prime}+3}}+\ldots\right)-\left(\frac{0}{3^{k^{\prime}}}+\sum_{m=k^{\prime}+1}^{\infty} \frac{\alpha_{m}\left(x_{n}\right)}{3^{m}}\right)}{\frac{2}{3^{k^{n}}}+\frac{1}{3^{k^{\prime \prime}}} \theta\left(y_{n}\right)-\frac{1}{3^{k^{n}}} \frac{3}{4}} \\
& =\frac{\frac{1}{3^{k^{\prime}}}\left(\frac{9}{4}-\theta\left(x_{n}\right)\right)}{\frac{1}{3^{k^{n}}}\left(\frac{5}{4}+\theta\left(y_{n}\right)\right)}=\frac{3^{k^{\prime \prime}}}{3^{k^{\prime}}} \frac{5+4\left(1-\theta\left(x_{n}\right)\right)}{5+4 \theta\left(y_{n}\right)}
\end{aligned}
$$

instead of (6.18), so we obtain $\theta_{3}$ and $\theta_{4}$ as

$$
\theta_{3}:=\lim _{l \rightarrow \infty}\left(1-\theta\left(x_{n_{l}}\right)\right), \quad \theta_{4}:=\lim _{l \rightarrow \infty} \theta\left(y_{n_{l}}\right) .
$$

We omit here the other details of the proof of statement (ii).

Now we are ready to prove the main result of the section.

Theorem 6.8. Let $X=C$ and $a$ be a point of $C$. The pretangent space $\Omega_{a, \tilde{r}}^{C}$ is unique for every normalizing sequence $\tilde{r}$ if and only if $a \in C^{1}$.

Proof. As has been shown, see Corollary 6.2, pretangent spaces are unique if $a \in C^{1}$. If $a \in C^{0} \backslash C^{2}$ then, by Lemma 6.5, the membership relation

$$
3 \in \bigcap_{\varepsilon \in \mathbf{R}_{+}^{*}}\left(C l\left({ }_{+} K_{\varepsilon} \cup{ }_{-} K_{\varepsilon}\right) \cap C l\left({ }_{1} K_{\varepsilon}\right)\right)
$$

holds. Consequently, by Theorem 5.4, $(C, a) \notin \mathfrak{U}$. Let $a$ belong to $C^{2}$. By Theorem $5.4(C, a) \notin \mathfrak{U}$ if there is a positive number belonging to $\bigcap_{\varepsilon \in \mathbf{R}_{+}^{*}}\left(C l\left({ }_{+} K_{\varepsilon} \cup{ }_{-} K_{\varepsilon}\right) \cap\right.$ $\left.C l\left({ }_{1} K_{\varepsilon}\right)\right)$. The last is possible if and only if there exist $m, n \in \mathbf{Z}$ and $\theta_{1}, \theta_{2}, \theta_{3}, \theta_{4} \in C$ such that

$$
3^{2 m} \frac{5+4 \theta_{1}}{5+4 \theta_{2}}=3^{2 n+1} \frac{5+4 \theta_{3}}{5+4 \theta_{4}},
$$

see Lemma 6.7. The direct calculation shows that the sextuple $\left(m, n, \theta_{1}, \theta_{2}, \theta_{3}, \theta_{4}\right)$ of numbers

$$
\begin{aligned}
& m=0, \quad n=0, \quad \theta_{1}=\frac{2}{3}+\frac{2}{3^{2}}, \quad \theta_{2}=0, \theta_{4}=1, \\
& \theta_{3}=1-\left(\frac{2}{3}+\frac{2}{3^{2}}+\sum_{j=0}^{\infty} \frac{2}{3^{3+4 j}}+\sum_{j=0}^{\infty} \frac{2}{3^{6+4 j}}\right)
\end{aligned}
$$

is a solution of equation (6.24). Thus $(C, a) \notin \mathfrak{U}$ for every $a \in C^{0}$.

Remark 6.9. In fact, solution (6.25) was obtained by the following way. For $m=n=\theta_{2}=0$ and $\theta_{4}=1$ equation (6.24) can be reduced to the form

$$
3 \theta_{1}+5\left(1-\theta_{3}\right)=\frac{15}{2}
$$

Applying to the last equation a variant of the so-called Greaddy algorithm we obtain the values of $\theta_{1}$ and $\theta_{4}$. 
Let us describe now the explicit form of the pretangent spaces $\Omega_{a, \tilde{r}}^{C}$ for $a \in C^{1}$ and $\tilde{r}=\left\{3^{-n}\right\}_{n \in \mathbf{N}}$. Define a set $C^{e}$ as the smallest subset of $\mathbf{R}$ which contains the Cantor set $C$ and satisfies the equality

$$
C^{e}=3^{n} C^{e}
$$

for every $n \in \mathbf{Z}$ where $3^{n} C^{e}:=\left\{3^{n} x: x \in C^{e}\right\}$. It follows from (6.2) that a real number $t$ belongs to $C^{e}$ if and only if $t$ has a base 3 expansion with the digits 0 and 2 only, i.e.,

$$
t=\sum_{j=-\infty}^{M} a_{n_{j}} 3^{j}
$$

with $M \in \mathbf{Z}$ and $a_{n_{j}} \in\{0,2\}$.

Proposition 6.10. Let $X=C$ be the Cantor set with the usual metric $|\cdot, \cdot|$ and let $\tilde{r}=\left\{3^{-n}\right\}_{n \in \mathbf{N}}$. For every $a \in C^{1}$ the pretangent space $\Omega_{0, \tilde{r}}^{X}$ is unique, tangent and isometric to $\left(C^{e},|\cdot, \cdot|\right)$.

Proof. Let $\tilde{X}_{a, \tilde{r}}$ be a maximal self-stable family for which $p\left(\tilde{X}_{a, \tilde{r}}\right)=\Omega_{a, \tilde{r}}^{X}$, see diagram (1.4). The relation $(C, a) \in \mathfrak{U}$ was proved above. It is clear that for every $a, b \in C^{1}$ there is $\varepsilon>0$ such that the sets

$$
C \cap] a-\varepsilon, a+\varepsilon[\text { and } C \cap] b-\varepsilon, b+\varepsilon[
$$

are isometric. Hence, without the loss of generality, put $a=0$. Then, as in the proof of Proposition 2.2, we see that for every $\tilde{x}=\left\{x_{n}\right\}_{n \in \mathbf{N}} \in \tilde{X}_{a, \tilde{r}}$ there exists a finite limit

$$
\lim _{n \rightarrow \infty} \frac{x_{n}}{3^{-n}}=c(\tilde{x})
$$

and that the function $f: \Omega_{a, \tilde{r}} \rightarrow \mathbf{R}^{+}$with

$$
f(\beta)=c(\tilde{x}) \quad \text { for } \quad \tilde{x} \in \beta \in \Omega_{a, \tilde{r}}
$$

is well-defined and distance-preserving. Consequently, $\Omega_{a, \tilde{r}}^{X}$ is isometric to $\left(C^{e},|\cdot, \cdot|\right)$ if the following two statements hold:

(i) $c(\tilde{x})$ belongs to $C^{e}$ for every $\tilde{x} \in \tilde{X}_{a, \tilde{r}}$;

(ii) For every $t \in C^{e}$ there is $\tilde{x} \in \tilde{X}_{a, \tilde{r}}$ such that $c(\tilde{x})=t$.

To prove statement (i) note that

$$
C^{e}=\bigcup_{i=0}^{\infty} 3^{i} C
$$

and that for every $t>0$ we have the equalities

$$
[0, t] \cap 3^{i} C=[0, t] \cap 3^{j} C
$$

if $i \wedge j \geq \log _{3} t$. Since $C$ is closed, equalities (6.31) and (6.30) imply that $C^{e}$ also is closed. Moreover, using (6.2) and (6.27) we see that

$$
\frac{x_{n}}{3^{-n}} \in C^{e}
$$

for all $x_{n} \in C$ and all $n \in \mathbf{N}$. Hence $c(\tilde{x})$ belongs to $C^{e}$ for every $\tilde{x} \in \tilde{X}_{a, \tilde{r}}$, that is statement (i) follows. 
Let $t$ be an arbitrary point of $C^{e}$. Then $3^{-n} t \in C$ if $n>M$, see (6.27). Write

$$
x_{n}:= \begin{cases}0 & \text { if } n \leq M \\ 3^{-n} t & \text { if } n>M\end{cases}
$$

for $n \in \mathbf{N}$ and define $\tilde{x}:=\left\{x_{n}\right\}_{n \in \mathbf{N}}$. It is clear that $c(\tilde{x})=t$, so statement (ii) is true.

It still remains to prove that $\Omega_{a, \tilde{r}}^{X}$ is tangent. Let $\left\{n_{k}\right\}_{k \in \mathbf{N}}$ be an infinite strictly increasing sequence of natural numbers and let $\tilde{r}^{\prime}:=\left\{3^{-n_{k}}\right\}_{k \in \mathbf{N}}$. As in the proof of statement (i) we see that the equivalence

$$
\left(\tilde{x}=\left\{x_{k}\right\}_{k \in \mathbf{N}} \in \tilde{X}_{a, \tilde{r}^{\prime}}\right) \Leftrightarrow\left(\lim _{k \rightarrow \infty} \frac{x_{k}}{3^{-n_{k}}} \in C^{e}\right)
$$

holds for every $\tilde{x} \in \tilde{X}$. By statement (ii) we have $f\left(\Omega_{a, \tilde{r}}^{X}\right)=C^{e}$, where $f$ is defined in (6.29). Consequently, a function $\mathrm{em}^{\prime}: \Omega_{a, \tilde{r}}^{X} \rightarrow \Omega_{a, \tilde{r}^{\prime}}$, see (1.4), is surjective. Hence, by Proposition $1.5, \Omega_{a, \tilde{r}}^{X}$ is tangent.

\section{Independence of uniqueness conditions}

The main goal of the present section is the proof of the logical independence of conditions (i) and (ii) of Theorem 2.4. We shall do it by construction of two examples of metric spaces such that the condition related to the corresponding example is true but another one is false.

Proposition 7.1. Condition (i) of Theorem 2.4 does not imply condition (ii) of this theorem.

As we already know the pointed Cantor set $(C, a)$ belongs to the class $\mathfrak{U}$ if and only if $a \in C^{1}$. Hence to prove Proposition 7.1 it is sufficient to find $a \in C \backslash C^{1}=C^{0}$ such that the pointed space $(C, a)$ satisfies condition (i) of Theorem 2.4. To this end we shall describe this condition in terms of the ternary expansion of the marked point $a \in C^{0}$, see (6.2).

Let $x$ be a point of the Cantor ternary set $C$. Define a sequence $\left\{\mathscr{R}_{x}(n)\right\}_{n \in \mathbf{N}}$ by the rule

$$
\mathscr{R}_{x}(n):= \begin{cases}\inf \left\{m-n: \alpha_{m} \neq \alpha_{n}, m>n\right\} & \text { if } \exists m>n: \alpha_{m} \neq \alpha_{n} \\ \infty & \text { if } \forall m>n: \alpha_{m}=\alpha_{n}\end{cases}
$$

i.e.,

$$
\begin{aligned}
& \mathscr{R}_{x}(n)=1 \Leftrightarrow\left(\alpha_{n} \neq \alpha_{n+1}\right), \\
& \mathscr{R}_{x}(n)=2 \Leftrightarrow\left(\alpha_{n}=\alpha_{n+1}\right) \text { and }\left(\alpha_{n+1} \neq \alpha_{n+2}\right),
\end{aligned}
$$

and so on.

Lemma 7.2. Let $X=C$ and $a$ be a point of the set $C^{0}$. Condition (i) of Theorem 2.4 holds at the point $a$ if and only if

$$
\limsup _{n \rightarrow \infty} \mathscr{R}_{a}(n)<\infty \text {. }
$$

Proof. Suppose

$$
\limsup _{n \rightarrow \infty} \mathscr{R}_{a}(n)=\infty
$$


We must prove that condition (i) of Theorem 2.4 does not hold. For this purpose, in accordance with Lemma 5.1, we will construct the sequences $\tilde{x}=\left\{x_{n}\right\}_{n \in \mathbf{N}} \in \tilde{X}$ and $\tilde{y}=\left\{y_{n}\right\}_{n \in \mathbf{N}} \in \tilde{X}$ such that

$$
x_{n}<a<y_{n} \quad \text { and } \quad \lim _{n \rightarrow \infty} x_{n}=\lim _{n \rightarrow \infty} y_{n}=a
$$

and

$$
\lim _{n \rightarrow \infty} \frac{a-x_{n}}{y_{n}-a}=1
$$

for all $n \in \mathbf{N}$.

Recall that $a \in C^{0}$. Consequently, both $\alpha_{m}=0$ and $\alpha_{m}=1$ are contained infinitely many times in representation (6.2). Hence, it follows from (7.1), (7.3) and from $a \in C^{0}$, that there is an infinite, strictly increasing sequence of natural numbers $m_{n}$ such that either

$$
\alpha_{m_{n}-1}(a)=1, \quad \alpha_{m_{n}}(a)=0 \quad \text { and } \quad \lim _{n \rightarrow \infty} \mathscr{R}_{a}\left(m_{n}\right)=\infty
$$

or

$$
\alpha_{m_{n}-1}(a)=0, \quad \alpha_{m_{n}}(a)=1 \quad \text { and } \quad \lim _{n \rightarrow \infty} \mathscr{R}_{a}\left(m_{n}\right)=\infty .
$$

We shall confine ourselves to the consideration of the case where relations (7.5) hold. It is sufficient because the isometry $f: C \rightarrow C, f(x)=1-x$, transfers the points satisfying (7.6) to the points satisfying (7.5) and conversely. Let us define the points $x_{n}, y_{n} \in C$ by the rules

$$
\begin{aligned}
& \alpha_{m}\left(x_{n}\right):= \begin{cases}\alpha_{m}(a) & \text { if } m \leq m_{n}-2, \\
0 & \text { if } m=m_{n}-1, \\
1 & \text { if } m \geq m_{n},\end{cases} \\
& \alpha_{m}\left(y_{n}\right):= \begin{cases}\alpha_{m}(a) & \text { if } m \leq m_{n}-1, \\
1 & \text { if } m \geq m_{n},\end{cases}
\end{aligned}
$$

where $\alpha_{m}\left(x_{n}\right)$ and $\alpha_{m}\left(y_{n}\right)$ are digits of the ternary representation of $x_{n}$ and $y_{n}$ respectively, see (6.2). It follows directly from (7.7), (7.5) that $x_{n}<a<y_{n}$ and that $\lim _{n \rightarrow \infty} x_{n}=a=\lim _{n \rightarrow \infty} y_{n}$ and

$$
\begin{aligned}
\frac{a-x_{n}}{y_{n}-a} & =\frac{\sum_{m=1}^{\infty} \frac{2 \alpha_{m}(a)}{3^{m}}-\sum_{m=1}^{\infty} \frac{2 \alpha_{m}\left(x_{n}\right)}{3^{m}}}{\sum_{m=1}^{\infty} \frac{2 \alpha_{m}\left(y_{n}\right)}{3^{m}}-\sum_{m=1}^{\infty} \frac{2 \alpha_{m}(a)}{3^{m}}} \\
& =\frac{\frac{2}{3^{m}-1}+\sum_{m=m_{n}}^{\infty} \frac{2 \alpha_{m}(a)}{3^{m}}-\sum_{m=m_{n}}^{\infty} \frac{2}{3^{m}}}{\sum_{m=m_{n}}^{\infty} \frac{2}{3^{m}}-\sum_{m=m_{n}}^{\infty} \frac{2 \alpha_{m}(a)}{3^{m}}} \\
& =\frac{\frac{1}{3^{m_{n}-1}}+\sum_{m=m_{n}}^{\infty} \frac{2 \alpha_{m}(a)}{3^{m}}}{\frac{1}{3^{m}-1}-\sum_{m=m_{n}}^{\infty} \frac{2 \alpha_{m}(a)}{3^{m}}}=\frac{1+\sum_{m=1}^{\infty} \frac{2 \alpha_{m+m_{n-1}}(a)}{3^{m}}}{1-\sum_{m=1}^{\infty} \frac{2 \alpha_{m+m_{n}-1}(a)}{3^{m}}} .
\end{aligned}
$$

Using (7.1) and the equality $\alpha_{m_{n}}(a)=0$ we see that

$$
\sum_{m=1}^{\infty} \frac{2 \alpha_{m+m_{n}-1}(a)}{3^{m}}=\sum_{m=\mathscr{R}_{a}\left(m_{m}\right)+1}^{\infty} \frac{2 \alpha_{m+m_{n}-1}(a)}{3^{m}} \leq\left(\frac{1}{3}\right)^{\mathscr{R}_{a}\left(m_{n}\right)}
$$

Consequently, (7.5) and (7.8) imply (7.4) so that, by Lemma 5.1, condition (i) of Theorem 2.4 does not hold. 
Suppose now inequality (7.2) holds and $\tilde{x}=\left\{x_{n}\right\}_{n \in \mathbf{N}} \in \tilde{X}, \tilde{y}=\left\{y_{n}\right\}_{n \in \mathbf{N}} \in \tilde{X}$ are sequences with $x_{n}<a<y_{n}, n \in \mathbf{N}$, and with

$$
\lim _{n \rightarrow \infty} x_{n}=\lim _{n \rightarrow \infty} y_{n}=a .
$$

We shall prove that the limit relation (7.4) does not hold. By Lemma 5.1 it implies condition (i) of Theorem 2.4. Let us define the quantities $k^{\prime}=k^{\prime}\left(x_{n}, a\right)$ and $k^{\prime \prime}=$ $k^{\prime \prime}\left(y_{n}, a\right)$ as

$$
k^{\prime}=\min \left\{m: \alpha_{m}\left(x_{n}\right) \neq \alpha_{m}(a)\right\}, \quad k^{\prime \prime}=\min \left\{m: \alpha_{m}\left(y_{n}\right) \neq \alpha_{m}(a)\right\} .
$$

Recall that triadic representation (6.2) is unique for the points of the Cantor set so that $k^{\prime}$ and $k^{\prime \prime}$ are correctly defined. The double inequality $x_{n}<a<y_{n}$ implies

$$
\left\{m: \alpha_{m}\left(x_{n}\right) \neq \alpha_{m}(a)\right\} \neq \emptyset \neq\left\{m: \alpha_{m}\left(y_{n}\right) \neq \alpha_{m}(a)\right\} .
$$

Note also that

$$
\left(x_{n}<a\right) \Rightarrow\left(\alpha_{k^{\prime}}\left(x_{n}\right)=0 \text { and } \alpha_{k^{\prime}}(a)=1\right)
$$

and similarly

$$
\left(y_{n}<a\right) \Rightarrow\left(\alpha_{k^{\prime \prime}}\left(x_{n}\right)=1 \text { and } \alpha_{k^{\prime \prime}}(a)=0\right),
$$

thus $k^{\prime} \neq k^{\prime \prime}$. We shall restrict ourselves to the case $k^{\prime}<k^{\prime \prime}$. The case $k^{\prime \prime}<k^{\prime}$ can be analyzed analogously.

Suppose that $k^{\prime \prime}=k^{\prime}+1$. Then using (7.10) and (7.11) we obtain

$$
\begin{aligned}
a-x_{n} & =\frac{2}{3^{k^{\prime}}}+\sum_{m=k^{\prime}+2}^{\infty} \frac{2 \alpha_{m}(a)}{3^{m}}-\sum_{m=k^{\prime}+1}^{\infty} \frac{2 \alpha_{m}\left(x_{n}\right)}{3^{m}} \\
& \geq \frac{2}{3^{k^{\prime}}}+\sum_{m=k^{\prime}+2}^{\infty} \frac{2 \alpha_{m}(a)}{3^{m}}-\frac{1}{3^{k^{\prime}}}=\frac{1}{3^{k^{\prime}}}+\sum_{m=k^{\prime}+2}^{\infty} \frac{2 \alpha_{m}(a)}{3^{m}}
\end{aligned}
$$

and

$$
\begin{aligned}
y_{n}-a & =\frac{2}{3^{k^{\prime}}+1}+\sum_{m=k^{\prime}+2}^{\infty} \frac{2 \alpha_{m}\left(y_{n}\right)}{3^{m}}-\sum_{m=k^{\prime}+2}^{\infty} \frac{2 \alpha_{m}(a)}{3^{m}} \\
& \leq \frac{2}{3^{k^{\prime}}+1}+\frac{1}{3^{k^{\prime}}+1}-\sum_{m=k^{\prime}+2}^{\infty} \frac{2 \alpha_{m}(a)}{3^{m}}=\frac{1}{3^{k^{\prime}}}-\sum_{m=k^{\prime}+2}^{\infty} \frac{2 \alpha_{m}(a)}{3^{m}} .
\end{aligned}
$$

Write for $n \in \mathbf{N}$

$$
t_{n}(a):=3^{n} \sum_{m=n+2}^{\infty} \frac{2 \alpha_{m}(a)}{3^{m}} .
$$

It follows form (7.12) and (7.13) that

$$
\frac{a-x_{n}}{y_{n}-a} \geq \frac{1+t_{k^{\prime}}(a)}{1-t_{k^{\prime}}(a)} \text {. }
$$

The simple estimations show that (7.2) holds if and only if there are constants $c_{1}, c_{2}$ such that

$$
0<c_{1} \leq 3^{n} \sum_{m=n}^{\infty} \frac{2 \alpha_{m}(a)}{3^{m}} \leq c_{2}<1
$$


for every $n=1,2, \ldots$ Hence, by the definition of $t_{n}(a)$, we obtain

$$
\frac{c_{1}}{9} \leq t_{n}(a) \leq \frac{c_{2}}{9}
$$

Since $\left.f(x)=\frac{1+x}{1-x}, x \in\right] 0,1[$, is an increasing function, inequalities (7.14) and (7.15) imply

$$
\frac{a-x_{n}}{y_{n}-a} \geq \frac{1+\frac{c_{1}}{9}}{1-\frac{c_{1}}{9}}>1 \text {. }
$$

Thus (7.4) does not hold.

In the simpler case where $k^{\prime \prime} \geq k^{\prime}+2$ the similar arguments yield

$$
\frac{a-x_{n}}{y_{n}-a} \geq 3
$$

that also contradicts (7.4).

Proof of Proposition 7.1. If we choose the point $a=\frac{1}{4}$ as marked point on $C$, then $\mathscr{R}_{a}(n)=1$ for sufficiently large $n$, see (7.1) and (6.6). Consequently, by Lemma 7.2, condition of Theorem 2.4 holds for the space $(C, a)$. Since $a=1 / 4 \in C^{0}$, Theorem 6.8 implies that condition (ii) of Theorem 2.4 does not hold with this $a$.

Proposition 7.3. Condition (ii) of Theorem 2.4 does not imply condition (i) of this theorem.

To prove Proposition 7.3 consider the following

Example 7.4. Let $\tilde{r}=\left\{r_{i}\right\}_{i \in \mathbf{N}}$ and $\tilde{\varepsilon}=\left\{\varepsilon_{i}\right\}_{i \in \mathbf{N}}$ be two strictly decreasing sequences of positive numbers such that

$$
r_{i+1}+\varepsilon_{i+1}<r_{i}
$$

for all $i \in \mathbf{N}$ and

$$
\lim _{i \rightarrow \infty} \frac{\varepsilon_{i+1}}{r_{i+1}}=\lim _{i \rightarrow \infty} \frac{r_{i+1}}{r_{i}}=0
$$

Define the pointed metric space $(X, a)$ with $X \subseteq \mathbf{R}$ and $a=0$ as

$$
X=\left\{r_{i}: i \in \mathbf{N}\right\} \cup\{0\} \cup\left\{-r_{i}-\varepsilon_{i}: i \in \mathbf{N}\right\},
$$

i.e., $X$ is the set consisting of the origin and the members of the sequences $\tilde{r}$ and $-\tilde{r}-\tilde{\varepsilon}$. It follows from (7.16) and (7.17) that in this space the equality

$$
\lim _{i \rightarrow \infty} \frac{A_{0}\left(r_{i}, k\right)}{r_{i}}=2
$$

holds for each $k>1$. Consequently, condition (i) of Theorem 2.4 does not hold for this $X$.

By Proposition 5.3 condition (ii) of Theorem 2.4 holds if and only if

$$
\bigcap_{\varepsilon \in \mathbf{R}_{+}^{*}}\left(C l\left({ }_{+} K_{\varepsilon} \cup_{-} K_{\varepsilon}\right) \cap C l\left({ }_{1} K_{\varepsilon}\right)\right) \subseteq\{0,1, \infty\} .
$$

Let us prove the last inclusion. Suppose that $\left\{x_{n}\right\}_{n \in \mathbf{N}},\left\{y_{n}\right\}_{n \in \mathbf{N}} \in \tilde{X}$ are sequences such that $x_{n}$ and $y_{n}$ are positive for all $n \in \mathbf{N}$ and $\lim _{n \rightarrow \infty} x_{n}=\lim _{n \rightarrow \infty} y_{n}=0$ and $\lim _{i \rightarrow \infty} \frac{x_{n}}{y_{n}}$ is finite. Then, for each $n \in \mathbf{N}$, there are $i, j \in \mathbf{N}$ such that

$$
x_{n}=r_{i} \quad \text { and } \quad y_{n}=r_{j} .
$$


These equalities and the second limit relation in (7.17) imply that

$$
\lim _{n \rightarrow \infty} \frac{x_{n}}{y_{n}} \quad \text { is either } 1 \text { or } 0 \text {. }
$$

Hence

$$
\bigcap_{\varepsilon \in \mathbf{R}_{+}^{*}} C l\left({ }_{+} K_{\varepsilon}\right) \subseteq\{0,1, \infty\} .
$$

Similarly, replacing $r_{n}$ by $r_{n}+\varepsilon_{n}$ we obtain

$$
\bigcap_{\varepsilon \in \mathbf{R}_{+}^{*}} C l\left({ }_{-} K_{\varepsilon}\right) \subseteq\{0,1, \infty\} .
$$

Since

$$
C l\left({ }_{+} K_{\varepsilon}\right) \cup C l\left({ }_{-} K_{\varepsilon}\right)=C l\left({ }_{+} K_{\varepsilon} \cup{ }_{-} K_{\varepsilon}\right),
$$

inclusions (7.19) and (7.20) implies (7.18). Thus condition (ii) of Theorem 2.4 holds.

Remark 7.5. It follows from (7.16) that 0 is local asymmetry point of $X$ in the previous example.

Acknowledgements. This paper was started in 2008 during a visit of the second author to the Mersin University (Turkey) under the support of the TÜBİTAKFellowships For Visiting Scientists Programme. The authors are very thankful to the anonymous referee for the interesting and productive refereeing.

\section{References}

[1] Ambrosio, L.: Metric space valued functions of bounded variation. - Ann. Sc. Norm. Super. Pisa Cl. Sci. (4) 17:3, 1990, 439-478.

[2] Ambrosio, L: Fine properties of sets of finite perimeter in doubling metric measure spaces. Set-Valued Anal. 10:2-3, 2002, 111-128.

[3] Ambrosio, L., and B. Kircheim: Rectifiable sets in metric and Banach spaces. - Math. Ann. 318:3, 2000, 527-555.

[4] Ambrosio, L., and B. Kircheim: Currents in mertic spaces. - Acta Math. 185:2, 2000, 1-80.

[5] Berger, M.: Géométrie. - Cedic, Paris, 1977, 1978; Nathan, Paris, 1977, 1978.

[6] Bourbaki, N.: General topology: Topological groups. Numbers and the groups and spaces related to them. - Izdat. Nauka, Moscow, 1969 (in Russian).

[7] Cheeger, J.: Differentiability of Lipschitz functions on metric measure spaces. - Geom. Funct. Anal. 9, 1999, 428-517.

[8] Collongwood, E. F., A. J. Lohwater: The theory of cluster sets. - Cambridge Univ. Press, Cambridge, 1966.

[9] Dovgoshey, O.: Tangent spaces to metric spaces and to their subspaces. - Ukr. Math. Visn. $5: 4,2008,470-487$.

[10] Dovgoshey, O., F. Abdullayev, and M. KüÇÜKaslan: Tangent metric spaces to convex sets on the plane. - Univ. Helsinki, Reports in Math. 481, 2008, 1-31.

[11] Dovgoshey, O., F. Abdullayev, and M. KüÇÜkaslan: Compactness and boundedness of tangent spaces to metric spaces. - Beiträge Algebra Geom. 51:2, 2010, 547-576.

[12] Dovgoshey, O., and D. Dordovskyi: Ultrametricity and metric betweenness in tangent spaces to metric spaces. - p-Adic Numbers, Ultrametric Analysis and Applications 2:2, 2010, 100-113. 
[13] Dovgoshey, O., and D. Dordovskyi: Ultrametricity of tangent spaces to metric spaces. Dopov. Nats. Akad. Nauk Ukr. 3, 2010, 19-23 (in Russian).

[14] Dovgoshey, O., and O. Martio: Tangent spaces to metric spaces. - Univ. Helsinki, Reports in Math. 480, 2008, 1-20.

[15] Hajlasz, P.: Sobolev spaces on an arbitrary metric space. - Potential Anal. 5, 1996, 403-415.

[16] Heinonen, J.: Lectures on analysis on metric spaces. - Springer-Verlag, NewYork, 2001.

[17] Heinonen, J., and P. Koskela: From local to global in quasiconformal structures. - Proc. Natl. Acad. Sci. USA 93, 1996, 554-556.

[18] Kelley, J. R.: General topology. - D. Van Nostrand Company, Princeton, 1965.

[19] Pontruagin, L. S.: Continuous groups. - The third corrected edition, Nauka, Moscow, 1979 (in Russian).

[20] Royden, H. L.: Real analysis. - The third edition, Macmillan Publ. Company, New York, 1988.

[21] Tномsом, B. S.: Symmetric properties of real functions. - Marcel Dekker, Inc., New York, 1994.

[22] Shanmugalingam, N.: Newtonian spaces: an extention of Sobolev spaces to metric measure spaces. - Rev. Mat. Iberoamericana 16, 2000, 243-279.

Received 11 November 2008

Revised received 9 September 2010 